\title{
Limitations of WRF land surface models for simulating land use and land cover change in Sub-Saharan Africa and development of an improved model (CLM-AF
} v. 1.0)

\author{
Timothy Glotfelty ${ }^{1}$, Diana Ramírez-Mejía ${ }^{2}$, Jared Bowden ${ }^{3}$, Adrian Ghilardi ${ }^{2}$, and J. Jason West ${ }^{1}$ \\ 1Department of Environmental Sciences and Engineering, University of North Carolina at Chapel Hill, Chapel Hill, \\ NC 27599, USA \\ 2Centre for Research in Environmental Geography, Universidad Nacional Autónoma de México, Morelia, 58190, \\ Mexico \\ 3Department of Applied Ecology, North Carolina State University, Raleigh, NC 27695, USA \\ Supplementary Material
}

\section{Addressing Inconsistencies in CLM-AF PFTs}

In the savanna category, corn is the fourth most abundant PFT indicating some misclassification with croplands. To eliminate this issue of misclassification, the corn contribution to the savanna category is ignored and the fifth most abundant PFT (broad leaf deciduous tropical trees) is used instead. In the broad leaf evergreen forest category, the third and fourth most abundant PFTs are deciduous tropical and temperate trees. To fix this misclassification, the contributions of each type of deciduous tree are added to their evergreen counterparts (i.e., tropical and temperate) to obtain an appropriate weighted total of tropical to temperate trees. After this, all tropical and temperate trees were assumed to be evergreen to match the MODIS category. In the mosaic cropland category, the fourth most abundant PFT is evenly divided between evergreen and deciduous tropical trees. This is addressed by summing the total deciduous and tropical tree amounts and assuming that all trees within this category were represented by evergreen tropical trees, since most mosaic cropland is in proximity to broadleaf evergreen forests.

\section{Additional Adjustments to LAI Broad Leaf Evergreen Tree Profiles}


Some additional adjustments were required for the monthly LAI profiles to ensure the best possible match to the satellite derived climatology. The broad leaf evergreen forest MODISIGBP category does not differentiate between tropical and temperate evergreen trees leading to an inconsistency with CLM. As mentioned in the previous section, the broad leaf evergreen forest category is split between tropical and temperate evergreen trees, however, the "one size fits all" approach to PFTs in WRF-CLM does not allow this ratio to change throughout the model domain. This is not reflective of actual PFT distributions as regions like the Congo contain only tropical evergreen trees, while regions like South Africa contain only temperate evergreen trees. To correct for the inconsistency, the broad leaf evergreen temperate trees were given the identical monthly LAI profile as tropical trees in the WW, WWN, and CW regions and the broad leaf evergreen tropical trees were given the identical monthly LAI profiles as the temperate trees in the SSD and SM regions.

\section{Subdivision of Bio-Climate Regions}

The central wet (CW) and northeast semi-dry (NESD) bioclimate regions are split into three sub-bioclimate regions. CW is split into central wet north (CWN) and central wet south (CWS) regions, north and south of the equator respectively. The southern portion of Angola that contains the savanna MODIS-IGBP category is further split from the CWS region (CWSA) to better represent the differences in LAI between tropical and subtropical grasses. The NESD region is also split into NESD north (NESDN) and NESD south (NESDS) regions containing the area within the NESD region north and south of $4^{\circ} \mathrm{N}$, respectively. To avoid an arbitrary discontinuity in LAI from occurring at $4^{\circ} \mathrm{N}$, the portion of the NESDN region that contains open shrubland around $4^{\circ} \mathrm{N}$ is further split off into a separate region (NESDSH) with its own LAI 
profiles. The central moist $(\mathrm{CM})$ region is also split into two sub-bioclimate regions, central mo

\section{Additional Details Regarding Moisture Variable Evaluation}

In most regions cloud fraction (CF) NMB and NME compared to MODIS are under 50\% across most LSMs (Fig. 8). The areas experiencing the greatest overpredictions and worst performance are those across the northern part of the simulation domain (i.e., ND, ED, NESD, EW, and WSD). This poor performance could result from multiple causes including domain boundary effects, errors in the cloud fraction parameterization, and poor coupling of cloud fraction parameterization to the cumulus parameterization. Similar CF performance occurs also occurs against CRU (Fig. S7) estimates.

The overprediction of precipitation (PRE) the EW region is likely the result of poorly resolved complex terrain in the Ethiopian Highlands at 36km grid spacing, as this poor performance is common to all WRF LSM simulations. Strong underpredictions in the LVW region are also consistent across LSMs. This is likely due inaccurate initialization of Lake Victoria's surface temperatures, which has been shown to substantially reduce simulated precipitation in the region (Argent et al., 2015). In contrast, the magnitude of the underpredictions in PRE within the East Africa regions varies more between LSMs. These regions experience the strongest underpredictions with the Noah LSM, likely as a result of insufficient atmospheric instability from the underpredicted surface temperatures. Some East Africa regions (e.g., SESD and EM) also experience considerable underpredictions in CLM-D, likely as a result of the inaccurate LAI seasonal profile underestimating the LAI during these regions' rainy season (JFD). This indicates that accurate seasonal LAI profiles are an important component to obtaining accurate rainfall predictions in these East African regions. The precipitation performance against TRMM observations discussed above (Fig. 8) are also 
generally comparable to GPCP and CRU estimates (Fig. S5), but TRMM error statistics are generally larger due to its greater horizontal resolution and spatial variability.ist north (CMN) and central moist south (CMS) north and south of $1^{\circ} \mathrm{N}$, respectively. 
Table S1: Percentage of Plant Functional Types Assigned to MODIS Land Use Categories in the Default CLM

\begin{tabular}{|c|c|c|c|c|c|c|c|c|c|c|c|c|c|c|c|c|}
\hline MODIS Land Use Category & 1 & 2 & 3 & 4 & 5 & 6 & 7 & 8 & 9 & 10 & 12 & 14 & 16 & 18 & 19 & 20 \\
\hline Bare soil & 25 & 5 & 50 & 25 & 26 & 20 & 20 & 20 & - & 20 & 15 & 15 & 90 & 50 & 60 & 80 \\
\hline Needle Leaf Evergreen Temperate Tree & 75 & - & - & - & 37 & - & - & - & - & - & - & - & - & - & - & - \\
\hline Needle Leaf Evergreen Boreal Tree & - & - & - & - & - & - & - & - & - & - & - & - & - & 13 & - & - \\
\hline Needle Leaf Deciduous Boreal Tree & - & - & 50 & - & - & - & - & - & - & - & - & - & - & 13 & - & - \\
\hline Broad Leaf Evergreen Tropical Tree & - & 95 & - & - & - & - & - & - & - & - & - & - & - & - & - & - \\
\hline Broad Leaf Evergreen Temperate Tree & - & - & - & - & - & - & - & - & 30 & - & - & - & - & - & - & - \\
\hline Broad Leaf Deciduous Tropical Tree & - & - & - & - & - & - & - & - & - & - & - & - & - & - & - & - \\
\hline Broad Leaf Deciduous Temperate Tree & - & - & - & 75 & 37 & - & - & - & - & - & - & - & - & - & - & - \\
\hline Broad Leaf Deciduous Boreal Tree & - & - & - & - & - & - & - & - & - & - & - & - & - & - & - & - \\
\hline Broad Leaf Evergreen Shrub & - & - & - & - & - & 80 & 50 & 80 & - & - & - & - & - & - & - & - \\
\hline Broad Leaf Deciduous Temperate Shrub & - & - & - & - & - & - & - & - & - & - & - & - & - & 24 & - & - \\
\hline Broad Leaf Deciduous Boreal Shrub & - & - & - & - & - & - & - & - & - & - & - & - & 10 & - & 20 & 10 \\
\hline C3 Artic Grass & - & - & - & - & - & - & - & - & - & - & - & - & - & - & 20 & 20 \\
\hline C3 Non-Artic Grass & - & - & - & - & - & - & - & - & - & 20 & - & - & - & - & - & - \\
\hline C4 Grass & - & - & - & - & - & - & 30 & - & 70 & 60 & - & 35 & - & - & - & - \\
\hline Corn & - & - & - & - & - & - & - & - & - & - & 85 & 50 & - & - & - & - \\
\hline Wheat & - & - & - & - & - & - & - & - & - & - & - & - & - & - & - & - \\
\hline
\end{tabular}

MODIS Land Use Categories: 1 - Evergreen Needle Leaf Forest, 2 - Evergreen Broad Leaf Forest; 3 - Deciduous Needle Leaf

Forest; 4 - Deciduous Broad Leaf Forest; 5 - Mixed Forest; 6 - Closed Shrublands; 7 - Open Shrublands; 8 - Woody Savanna; 9 -

Savannas; 10 - Grasslands; 12 - Croplands; 14 -Cropland/Natural Mosaic; 16 - Barren/ Sparsely Vegetated; 18 - Wooded Tundra; 19

- Mixed Tundra; 20 - Barren Tundra 
Table S2: Default CLM Monthly Leaf Area Index Profiles

\begin{tabular}{|c|c|c|c|c|c|c|c|c|c|c|c|c|}
\hline Month & 1 & 2 & 3 & 4 & 5 & 6 & 7 & 8 & 9 & 10 & 11 & 12 \\
\hline Needle Leaf Evergreen Temperate Tree & 4.1 & 4.2 & 4.6 & 4.8 & 4.9 & 5.0 & 4.8 & 4.7 & 4.6 & 4.2 & 4.0 & 4.0 \\
\hline Needle Leaf Evergreen Boreal Tree & 4.1 & 4.2 & 4.6 & 4.8 & 4.9 & 5.0 & 4.8 & 4.7 & 4.6 & 4.2 & 4.0 & 4.0 \\
\hline Needle Leaf Deciduous Boreal Tree & 0.0 & 0.0 & 0.0 & 0.6 & 1.2 & 2.0 & 2.6 & 1.7 & 1.0 & 0.5 & 0.2 & 0.0 \\
\hline Broad Leaf Evergreen Tropical Tree & 4.5 & 4.5 & 4.5 & 4.5 & 4.5 & 4.5 & 4.5 & 4.5 & 4.5 & 4.5 & 4.5 & 4.5 \\
\hline Broad Leaf Evergreen Temperate Tree & 4.5 & 4.5 & 4.5 & 4.5 & 4.5 & 4.5 & 4.5 & 4.5 & 4.5 & 4.5 & 4.5 & 4.5 \\
\hline Broad Leaf Deciduous Tropical Tree & 0.8 & 0.7 & 0.4 & 0.5 & 0.5 & 0.7 & 1.7 & 3.0 & 2.5 & 1.6 & 1.0 & 1.0 \\
\hline Broad Leaf Deciduous Temperate Tree & 0.0 & 0.0 & 0.3 & 1.2 & 3.0 & 4.7 & 4.5 & 3.4 & 1.2 & 0.3 & 0.0 & 0.0 \\
\hline Broad Leaf Deciduous Boreal Tree & 0.0 & 0.0 & 0.3 & 1.2 & 3.0 & 4.7 & 4.5 & 3.4 & 1.2 & 0.3 & 0.0 & 0.0 \\
\hline Broad Leaf Evergreen Shrub & 1.0 & 1.0 & 1.0 & 1.0 & 1.0 & 1.0 & 1.0 & 1.0 & 1.0 & 1.0 & 1.0 & 1.0 \\
\hline Broad Leaf Deciduous Temperate Shrub & 0.9 & 0.8 & 0.2 & 0.2 & 0.0 & 0.0 & 0.0 & 0.2 & 0.4 & 0.5 & 0.6 & 0.8 \\
\hline Broad Leaf Deciduous Boreal Shrub & 0.0 & 0.0 & 0.0 & 0.0 & 0.0 & 0.2 & 1.4 & 1.2 & 0.0 & 0.0 & 0.0 & 0.0 \\
\hline C3 Artic Grass & 0.4 & 0.5 & 0.6 & 0.7 & 1.2 & 3.0 & 3.5 & 1.5 & 0.7 & 0.6 & 0.5 & 0.4 \\
\hline C3 Non-Artic Grass & 0.0 & 0.0 & 0.0 & 0.0 & 0.0 & 0.2 & 1.4 & 1.2 & 0.0 & 0.0 & 0.0 & 0.0 \\
\hline C4 Grass & 0.4 & 0.5 & 0.6 & 0.7 & 1.2 & 3.0 & 3.5 & 1.5 & 0.7 & 0.6 & 0.5 & 0.4 \\
\hline Corn & 0.0 & 0.0 & 0.0 & 0.0 & 1.0 & 2.0 & 3.0 & 3.0 & 1.5 & 0.0 & 0.0 & 0.0 \\
\hline Wheat & 0.0 & 0.0 & 0.0 & 0.0 & 1.0 & 2.0 & 3.0 & 3.0 & 1.5 & 0.0 & 0.0 & 0.0 \\
\hline
\end{tabular}


Table S3: MODIS-IGBP Land Cover Category Description (Friedl et al., 2002)

\begin{tabular}{|c|c|}
\hline MODIS-IGBP Land Cover Category & Description \\
\hline Evergreen Broad Leaf Forests & $\begin{array}{l}\text { Lands dominated by broad leaf woody } \\
\text { vegetation with a percentage cover }>60 \% \text { and } \\
\text { height exceeding } 2 \mathrm{~m} \text {. Almost all trees and } \\
\text { shrubs remain green year round. Canopy is } \\
\text { never without foliage. }\end{array}$ \\
\hline Closed Shrubland & $\begin{array}{l}\text { Lands with woody vegetation less than } 2 \mathrm{~m} \\
\text { tall and with shrub cover }>60 \% \text {. The shrub } \\
\text { cover be either evergreen or deciduous. }\end{array}$ \\
\hline Open Shrubland & $\begin{array}{c}\text { Lands with woody vegetation less than } 2 \mathrm{~m} \\
\text { tall and with shrub canopy cover between } \\
10 \% \text { and } 60 \% \text {. The shrub foliage can be either } \\
\text { evergreen or deciduous. }\end{array}$ \\
\hline Woody Savanna & $\begin{array}{c}\text { Lands with herbaceous and other understory } \\
\text { systems, with forest canopy cover between } \\
30 \% \text { and } 60 \% \text {. The forest cover height } \\
\text { exceeds } 2 \mathrm{~m} \text {. }\end{array}$ \\
\hline Savanna & $\begin{array}{l}\text { Lands with herbaceous and other understory } \\
\text { systems, with forest and canopy cover } \\
\text { between } 10 \text { and } 30 \% \text {. The forest cover } \\
\text { exceeds } 2 \mathrm{~m} \text {. }\end{array}$ \\
\hline Grasslands & $\begin{array}{l}\text { Land with herbaceous types of cover. Tree } \\
\text { and shrub cover is less than } 10 \% \text {. }\end{array}$ \\
\hline Cropland & $\begin{array}{l}\text { Lands covered with temporary crops followed } \\
\text { by harvest and bare soil periods (e.g., single } \\
\text { and multiple cropping systems). Note that } \\
\text { perennial woody crops will be classified as } \\
\text { the appropriate forest of shrub land cover } \\
\text { type. }\end{array}$ \\
\hline Mosaic Cropland & $\begin{array}{c}\text { Lands with a mosaic of croplands, forests, } \\
\text { shrubland, and grasslands in which no one } \\
\text { component comprises more than } 60 \% \text { of the } \\
\text { landscape. }\end{array}$ \\
\hline
\end{tabular}


Table S4: Updated CLM Monthly Leaf Area Index Profiles for Broad Leaf Evergreen Tropical Trees

\begin{tabular}{|c|c|c|c|c|c|c|c|c|c|c|c|c|}
\hline Region & Jan. & Feb. & Mar. & Apr. & May & Jun. & Jul. & Aug. & Sep. & Oct. & Nov. & Dec. \\
\hline EW & 4.4 & 4.5 & 4.7 & 5.1 & 5.4 & 5.3 & 5.2 & 5.2 & 5.3 & 5.4 & 5.2 & 4.7 \\
\hline WW & 4.9 & 5.2 & 5.3 & 5.6 & 5.6 & 5.7 & 5.7 & 5.8 & 5.7 & 5.6 & 5.4 & 5.0 \\
\hline $\mathrm{CW}(\mathrm{N}, \mathrm{S}, \mathrm{SA})$ & 5.3 & 5.7 & 5.8 & 5.9 & 5.6 & 5.1 & 5.0 & 5.2 & 5.7 & 5.8 & 5.7 & 5.4 \\
\hline WWN & 3.3 & 3.5 & 3.7 & 4.2 & 4.3 & 4.2 & 4.3 & 4.5 & 4.5 & 4.3 & 4.3 & 3.9 \\
\hline $\mathrm{CM}(\mathrm{S})$ & 5.4 & 4.9 & 5.0 & 5.6 & 6.0 & 5.7 & 5.2 & 5.2 & 5.5 & 5.7 & 5.9 & 5.7 \\
\hline LVW & 4.8 & 4.5 & 4.6 & 5.1 & 5.4 & 5.3 & 5.0 & 5.0 & 5.2 & 5.2 & 5.3 & 5.2 \\
\hline $\mathrm{EM}^{60}$ & 3.1 & 3.2 & 3.3 & 3.4 & 3.4 & 2.9 & 2.6 & 2.6 & 2.6 & 2.5 & 2.5 & 2.8 \\
\hline MAD & 5.8 & 5.8 & 5.7 & 5.1 & 4.6 & 4.3 & 4.3 & 4.7 & 5.4 & 5.7 & 5.7 & 5.7 \\
\hline
\end{tabular}

${ }^{60}$ : Profile generated using $60 \%$ of grid cell threshold 
Table S5: Updated CLM Monthly Leaf Area Index Profiles for Broad Leaf Evergreen Temperate Trees

\begin{tabular}{ccccccccccccc}
\hline Region & Jan. & Feb. & Mar. & Apr. & May. & Jun. & Jul. & Aug. & Sep. & Oct. & Nov. & Dec. \\
\hline EW & 4.4 & 4.6 & 4.9 & 5.3 & 5.4 & 5.2 & 5.2 & 5.3 & 5.4 & 5.4 & 5.2 & 4.7 \\
CM (S) & 3.3 & 3.4 & 3.4 & 3.4 & 3.3 & 3.2 & 3.0 & 2.9 & 2.8 & 2.8 & 3.0 & 3.2 \\
LVW & 5.0 & 4.9 & 5.0 & 5.2 & 5.2 & 5.0 & 4.9 & 5.0 & 5.0 & 5.0 & 5.1 & 5.0 \\
EM & 3.5 & 3.6 & 3.5 & 3.3 & 3.0 & 2.5 & 2.2 & 2.3 & 2.5 & 2.4 & 2.7 & 3.1 \\
MAD & 5.0 & 5.1 & 4.8 & 4.3 & 3.9 & 3.6 & 3.6 & 3.7 & 4.4 & 4.9 & 5.0 & 4.9 \\
SSD & 2.3 & 2.1 & 2.0 & 1.6 & 1.5 & 1.4 & 1.4 & 1.5 & 1.8 & 2.1 & 2.3 & 2.3 \\
SM & 2.9 & 2.9 & 2.6 & 2.2 & 1.7 & 1.5 & 1.3 & 1.4 & 1.5 & 1.7 & 2.2 & 2.7 \\
\hline
\end{tabular}

${ }^{60}$ : Profile generated using $60 \%$ of grid cell threshold 
Table S6: Updated CLM Monthly Leaf Area Index Profiles for Broad Leaf Deciduous Tropical Trees

\begin{tabular}{|c|c|c|c|c|c|c|c|c|c|c|c|c|}
\hline Region & Jan. & Feb. & Mar. & Apr. & May & Jun. & Jul. & Aug. & Sep. & Oct. & Nov. & Dec. \\
\hline WSD & 0.6 & 0.6 & 0.5 & 0.6 & 0.7 & 1.1 & 1.6 & 1.9 & 1.9 & 1.6 & 1.0 & 0.7 \\
\hline NESD (N) & 0.7 & 0.7 & 0.7 & 0.7 & 0.9 & 1.2 & 1.7 & 1.9 & 1.9 & 1.5 & 1.1 & 0.8 \\
\hline $\operatorname{NESD}(\mathrm{S}, \mathrm{SH})^{60}$ & 2.1 & 2.0 & 1.9 & 2.2 & 2.3 & 2.1 & 2.0 & 1.8 & 1.5 & 1.4 & 1.8 & 2.1 \\
\hline EW & 0.3 & 0.3 & 0.3 & 0.3 & 0.3 & 0.4 & 0.8 & 1.1 & 1.2 & 0.9 & 0.6 & 0.4 \\
\hline WM & 1.3 & 1.3 & 1.5 & 1.8 & 2.0 & 2.3 & 2.6 & 2.8 & 2.8 & 2.6 & 2.1 & 1.7 \\
\hline WW & 2.3 & 2.2 & 2.2 & 2.3 & 2.5 & 3.0 & 3.4 & 3.6 & 3.6 & 3.6 & 3.3 & 2.6 \\
\hline $\mathrm{CW}(\mathrm{N})$ & 3.1 & 3.3 & 3.4 & 3.6 & 3.6 & 3.6 & 3.6 & 3.8 & 3.9 & 3.7 & 3.5 & 3.3 \\
\hline $\mathrm{CW}(\mathrm{S}, \mathrm{SA})$ & 2.9 & 2.9 & 3.0 & 2.7 & 1.8 & 1.3 & 1.0 & 1.1 & 1.6 & 2.1 & 2.7 & 2.9 \\
\hline SESD & 2.6 & 2.7 & 2.7 & 2.7 & 2.3 & 1.9 & 1.8 & 1.6 & 1.5 & 1.6 & 1.9 & 2.4 \\
\hline MAD & 1.9 & 2.1 & 2.1 & 1.7 & 1.2 & 0.8 & 0.7 & 0.7 & 0.7 & 0.7 & 0.8 & 1.3 \\
\hline $\mathrm{SSD}^{60}$ & 1.5 & 1.5 & 1.4 & 1.1 & 0.7 & 0.4 & 0.3 & 0.4 & 0.4 & 0.5 & 0.8 & 1.2 \\
\hline $\mathrm{SM}^{60}$ & 3.5 & 3.7 & 3.6 & 3.3 & 3.1 & 2.9 & 2.7 & 2.7 & 2.8 & 3.0 & 3.2 & 3.4 \\
\hline
\end{tabular}

${ }^{60}$ : Profile generated using $60 \%$ of grid cell threshold 
Table S7: Updated CLM Monthly Leaf Area Index Profiles for Broad Leaf Deciduous Temperate Shrub

\begin{tabular}{|c|c|c|c|c|c|c|c|c|c|c|c|c|}
\hline Region & Jan. & Feb. & Mar. & Apr. & May & Jun. & Jul. & Aug. & Sep. & Oct. & Nov. & Dec. \\
\hline $\mathrm{ED}^{60}$ & 0.3 & 0.2 & 0.2 & 0.4 & 0.5 & 0.4 & 0.2 & 0.2 & 0.2 & 0.4 & 0.5 & 0.5 \\
\hline $\mathrm{WSD}^{60}$ & 0.4 & 0.4 & 0.4 & 0.4 & 0.4 & 0.7 & 1.3 & 1.7 & 1.7 & 1.3 & 0.7 & 0.5 \\
\hline $\operatorname{NESD}(\mathrm{N})$ & 0.3 & 0.2 & 0.3 & 0.3 & 0.6 & 1.2 & 1.7 & 1.9 & 2.0 & 2.0 & 1.5 & 0.7 \\
\hline NESD (S) & 0.4 & 0.4 & 0.4 & 0.9 & 1.2 & 0.7 & 0.5 & 0.4 & 0.4 & 0.9 & 1.1 & 0.8 \\
\hline $\operatorname{NESD}(\mathrm{SH})^{60}$ & 0.3 & 0.3 & 0.3 & 0.4 & 0.6 & 0.4 & 0.3 & 0.3 & 0.3 & 0.4 & 0.5 & 0.4 \\
\hline $\mathrm{CW}(\mathrm{S})^{60}$ & 0.5 & 0.6 & 0.7 & 0.7 & 0.5 & 0.4 & 0.3 & 0.3 & 0.3 & 0.3 & 0.3 & 0.4 \\
\hline $\mathrm{CW}(\mathrm{SA})^{60}$ & 0.8 & 1.0 & 1.1 & 1.1 & 0.9 & 0.7 & 0.5 & 0.5 & 0.5 & 0.5 & 0.6 & 0.8 \\
\hline SESD $^{60}$ & 0.7 & 0.7 & 0.6 & 0.6 & 0.4 & 0.3 & 0.2 & 0.2 & 0.2 & 0.3 & 0.4 & 0.6 \\
\hline $\mathrm{MAD}^{60}$ & 1.0 & 1.1 & 1.1 & 1.0 & 0.8 & 0.6 & 0.5 & 0.4 & 0.4 & 0.4 & 0.5 & 0.7 \\
\hline $\mathrm{SD}^{60}$ & 0.3 & 0.3 & 0.2 & 0.2 & 0.2 & 0.3 & 0.3 & 0.4 & 0.4 & 0.4 & 0.3 & 0.3 \\
\hline $\mathrm{SSD}^{60}$ & 0.5 & 0.6 & 0.6 & 0.5 & 0.3 & 0.2 & 0.2 & 0.2 & 0.2 & 0.3 & 0.4 & 0.5 \\
\hline $\mathrm{SM}^{60}$ & 0.9 & 0.9 & 0.8 & 0.6 & 0.4 & 0.3 & 0.3 & 0.3 & 0.3 & 0.4 & 0.6 & 0.8 \\
\hline
\end{tabular}

${ }^{60}$ : Profile generated using $60 \%$ of grid cell threshold 
Table S8: Updated CLM Monthly Leaf Area Index Profiles for C3 Non-Artic Grass

\begin{tabular}{|c|c|c|c|c|c|c|c|c|c|c|c|c|}
\hline Region & Jan. & Feb. & Mar. & Apr. & May & Jun. & Jul. & Aug. & Sep. & Oct. & Nov. & Dec. \\
\hline $\mathrm{WSD}^{60}$ & 0.6 & 0.5 & 0.5 & 0.5 & 0.8 & 1.2 & 1.6 & 1.8 & 1.9 & 1.6 & 0.9 & 0.7 \\
\hline $\operatorname{NESD}(\mathrm{N})$ & 0.4 & 0.4 & 0.4 & 0.6 & 0.8 & 0.9 & 1.3 & 1.6 & 1.6 & 1.2 & 0.8 & 0.5 \\
\hline $\operatorname{NESD}(\mathrm{S})$ & 1.4 & 1.6 & 1.6 & 1.6 & 1.2 & 0.8 & 0.6 & 0.6 & 0.5 & 0.5 & 0.6 & 0.9 \\
\hline $\operatorname{NESD}(\mathrm{SH})^{60}$ & 1.0 & 0.9 & 0.8 & 1.2 & 1.3 & 1.0 & 0.8 & 0.9 & 0.9 & 0.9 & 1.2 & 1.2 \\
\hline EW & 0.5 & 0.4 & 0.4 & 0.5 & 0.6 & 0.8 & 1.3 & 1.8 & 1.9 & 1.5 & 1.0 & 0.6 \\
\hline $\mathrm{WW}^{60}$ & 1.0 & 1.0 & 1.1 & 1.4 & 1.6 & 2.1 & 2.4 & 2.5 & 2.6 & 2.4 & 1.8 & 1.2 \\
\hline $\mathrm{CW}(\mathrm{N})^{60}$ & 1.4 & 1.3 & 1.5 & 2.1 & 2.6 & 2.7 & 2.7 & 2.7 & 2.6 & 2.6 & 2.3 & 1.8 \\
\hline CW (S) & 1.8 & 1.8 & 1.8 & 1.8 & 1.7 & 1.3 & 1.1 & 1.2 & 1.5 & 1.8 & 1.9 & 1.9 \\
\hline CW (SA) & 1.4 & 1.5 & 1.6 & 1.5 & 1.2 & 0.8 & 0.6 & 0.6 & 0.6 & 0.8 & 1.1 & 1.3 \\
\hline $\mathrm{WWN}^{60}$ & 0.5 & 0.5 & 0.7 & 1.3 & 2.0 & 2.3 & 2.3 & 2.3 & 2.3 & 2.2 & 1.5 & 0.8 \\
\hline $\mathrm{CM}(\mathrm{N})^{60}$ & 1.0 & 0.9 & 0.9 & 1.3 & 1.6 & 1.7 & 1.7 & 1.7 & 1.6 & 1.4 & 1.4 & 1.2 \\
\hline $\mathrm{CM}(\mathrm{S})$ & 1.6 & 1.6 & 1.7 & 1.8 & 1.6 & 1.1 & 0.8 & 0.8 & 0.8 & 0.7 & 0.9 & 1.3 \\
\hline LVW & 1.4 & 1.2 & 1.3 & 1.5 & 1.8 & 1.7 & 1.6 & 1.5 & 1.5 & 1.4 & 1.5 & 1.5 \\
\hline EM & 1.5 & 1.6 & 1.6 & 1.5 & 1.2 & 1.0 & 0.8 & 0.7 & 0.7 & 0.7 & 0.9 & 1.2 \\
\hline SESD & 1.6 & 1.6 & 1.6 & 1.3 & 1.0 & 0.7 & 0.6 & 0.5 & 0.6 & 0.6 & 0.8 & 1.3 \\
\hline MAD & 1.3 & 1.6 & 1.6 & 1.5 & 1.1 & 0.9 & 0.7 & 0.6 & 0.6 & 0.6 & 0.8 & 1.0 \\
\hline $\mathrm{SD}^{60}$ & 0.3 & 0.3 & 0.4 & 0.3 & 0.3 & 0.3 & 0.3 & 0.3 & 0.3 & 0.3 & 0.3 & 0.3 \\
\hline SSD & 1.0 & 1.2 & 1.1 & 0.9 & 0.6 & 0.4 & 0.4 & 0.3 & 0.3 & 0.4 & 0.6 & 0.8 \\
\hline SM & 1.8 & 1.9 & 1.6 & 1.2 & 0.7 & 0.5 & 0.4 & 0.4 & 0.5 & 0.8 & 1.2 & 1.6 \\
\hline
\end{tabular}

${ }^{60}$ : Profile generated using $60 \%$ of grid cell threshold 
Table S9: Updated CLM Monthly Leaf Area Index Profiles for C4 Grass

\begin{tabular}{ccccccccccccc}
\hline Region & Jan. & Feb. & Mar. & Apr. & May & Jun. & Jul. & Aug. & Sep. & Oct. & Nov. & Dec. \\
\hline ND $^{60}$ & 0.2 & 0.1 & 0.1 & 0.1 & 0.1 & 0.2 & 0.3 & 0.4 & 0.5 & 0.3 & 0.2 & 0.2 \\
WSD & 0.4 & 0.4 & 0.4 & 0.4 & 0.5 & 0.9 & 1.3 & 1.7 & 1.7 & 1.3 & 0.8 & 0.5 \\
NESD (N) & 0.4 & 0.3 & 0.3 & 0.4 & 0.6 & 1.0 & 1.5 & 1.9 & 1.9 & 1.5 & 0.9 & 0.6 \\
NESD (S) & 1.2 & 1.0 & 1.0 & 1.3 & 1.4 & 1.2 & 1.1 & 0.9 & 0.8 & 0.9 & 1.2 & 1.3 \\
NESD (SH) & 0.3 & 0.3 & 0.2 & 0.4 & 0.4 & 0.3 & 0.4 & 0.5 & 0.4 & 0.4 & 0.4 & 0.4 \\
EW & 0.6 & 0.4 & 0.5 & 0.8 & 1.4 & 2.0 & 2.3 & 2.6 & 2.5 & 2.2 & 1.6 & 1.0 \\
WM & 0.5 & 0.6 & 0.6 & 0.9 & 1.2 & 1.5 & 1.8 & 2.0 & 2.0 & 1.9 & 1.2 & 0.7 \\
WW & 0.7 & 0.7 & 0.9 & 1.1 & 1.4 & 1.8 & 2.1 & 2.2 & 2.3 & 2.1 & 1.4 & 0.9 \\
CW (N) & 0.5 & 0.5 & 0.6 & 0.8 & 1.1 & 1.7 & 2.2 & 2.3 & 2.4 & 2.0 & 1.2 & 0.6 \\
CW (S) & 2.1 & 2.1 & 2.2 & 2.3 & 2.0 & 1.4 & 1.1 & 1.2 & 1.4 & 1.6 & 1.9 & 2.1 \\
CW (SA) & 1.5 & 1.7 & 1.8 & 1.7 & 1.3 & 0.9 & 0.7 & 0.7 & 0.7 & 0.8 & 1.1 & 1.5 \\
WWN & 0.5 & 0.6 & 0.7 & 1.0 & 1.5 & 1.8 & 2.0 & 2.1 & 2.1 & 2.1 & 1.4 & 0.8 \\
CM (N) & 0.6 & 0.6 & 0.6 & 0.9 & 1.4 & 2.0 & 2.3 & 2.5 & 2.2 & 1.7 & 1.1 & 0.7 \\
CM (S) & 1.7 & 1.7 & 1.7 & 1.7 & 1.6 & 1.2 & 1.1 & 0.9 & 0.8 & 0.8 & 1.1 & 1.5 \\
LWW & 0.8 & 0.7 & 0.8 & 1.1 & 1.3 & 1.3 & 1.3 & 1.3 & 1.2 & 1.2 & 1.2 & 0.9 \\
EM & 1.9 & 2.1 & 2.2 & 2.2 & 1.8 & 1.3 & 1.0 & 0.8 & 0.7 & 0.7 & 0.8 & 1.2 \\
SESD & 1.8 & 1.9 & 1.9 & 1.6 & 1.1 & 0.8 & 0.7 & 0.6 & 0.6 & 0.6 & 0.8 & 1.4 \\
MAD & 1.5 & 1.6 & 1.6 & 1.3 & 1.0 & 0.7 & 0.6 & 0.6 & 0.6 & 0.6 & 0.7 & 1.1 \\
SSD & 1.1 & 1.2 & 1.1 & 1.0 & 0.7 & 0.5 & 0.4 & 0.4 & 0.4 & 0.4 & 0.6 & 0.9 \\
\hline
\end{tabular}

${ }^{60}$ : Profile generated using $60 \%$ of grid cell threshold 
Table S10: Updated CLM Monthly Leaf Area Index Profiles for Corn

\begin{tabular}{ccccccccccccc}
\hline Region & Jan. & Feb. & Mar. & Apr. & May & Jun. & Jul. & Aug. & Sep. & Oct. & Nov. & Dec. \\
\hline ND & 0.1 & 0.1 & 0.1 & 0.1 & 0.1 & 0.2 & 0.2 & 0.3 & 0.3 & 0.2 & 0.1 & 0.1 \\
WSD & 0.2 & 0.2 & 0.1 & 0.2 & 0.2 & 0.3 & 0.4 & 0.6 & 0.6 & 0.5 & 0.3 & 0.2 \\
NESD (N) & 0.2 & 0.2 & 0.3 & 0.5 & 0.6 & 0.5 & 0.5 & 0.6 & 0.6 & 0.5 & 0.5 & 0.3 \\
NESD (S, SH) & 0.4 & 0.4 & 0.4 & 0.7 & 1.0 & 1.0 & 0.9 & 1.0 & 0.9 & 0.7 & 0.6 & 0.5 \\
EW ${ }^{60}$ & 0.4 & 0.5 & 0.5 & 0.6 & 0.6 & 0.6 & 0.8 & 1.2 & 1.3 & 1.1 & 0.7 & 0.5 \\
WM & 0.6 & 0.6 & 0.7 & 0.9 & 1.1 & 1.4 & 1.7 & 1.8 & 1.9 & 1.7 & 1.3 & 0.9 \\
WW & 0.6 & 0.8 & 1.1 & 1.6 & 1.9 & 2.0 & 2.2 & 2.2 & 2.2 & 2.3 & 1.5 & 0.8 \\
CW (N) & 0.9 & 1.0 & 1.2 & 1.7 & 2.3 & 2.6 & 2.7 & 2.6 & 2.6 & 2.5 & 1.8 & 1.1 \\
WWN & 0.7 & 0.8 & 1.0 & 1.3 & 1.7 & 1.9 & 2.0 & 2.1 & 2.1 & 2.0 & 1.6 & 1.0 \\
CM (N) & 0.6 & 0.5 & 0.7 & 1.4 & 2.2 & 2.5 & 2.5 & 2.6 & 2.6 & 2.2 & 1.7 & 1.0 \\
CM (S) & 1.6 & 1.4 & 1.4 & 1.7 & 1.9 & 1.4 & 1.0 & 0.9 & 1.1 & 1.5 & 1.8 & 1.8 \\
LVW & 1.1 & 1.0 & 1.1 & 1.5 & 1.8 & 1.7 & 1.6 & 1.6 & 1.5 & 1.5 & 1.5 & 1.2 \\
SESD & 1.8 & 1.9 & 1.9 & 1.7 & 1.3 & 0.9 & 0.8 & 0.6 & 0.6 & 0.6 & 0.8 & 1.3 \\
SSD & 0.6 & 0.6 & 0.6 & 0.5 & 0.4 & 0.5 & 0.7 & 0.8 & 0.7 & 0.5 & 0.4 & 0.5 \\
SM ${ }^{60}$ & 2.1 & 2.2 & 2.0 & 1.7 & 1.3 & 0.9 & 0.8 & 0.8 & 0.8 & 1.2 & 1.7 & 2.0 \\
\hline
\end{tabular}

${ }^{60}$ : Profile generated using $60 \%$ of grid cell threshold 
Table S11. Dinamica EGO Explanatory Variables

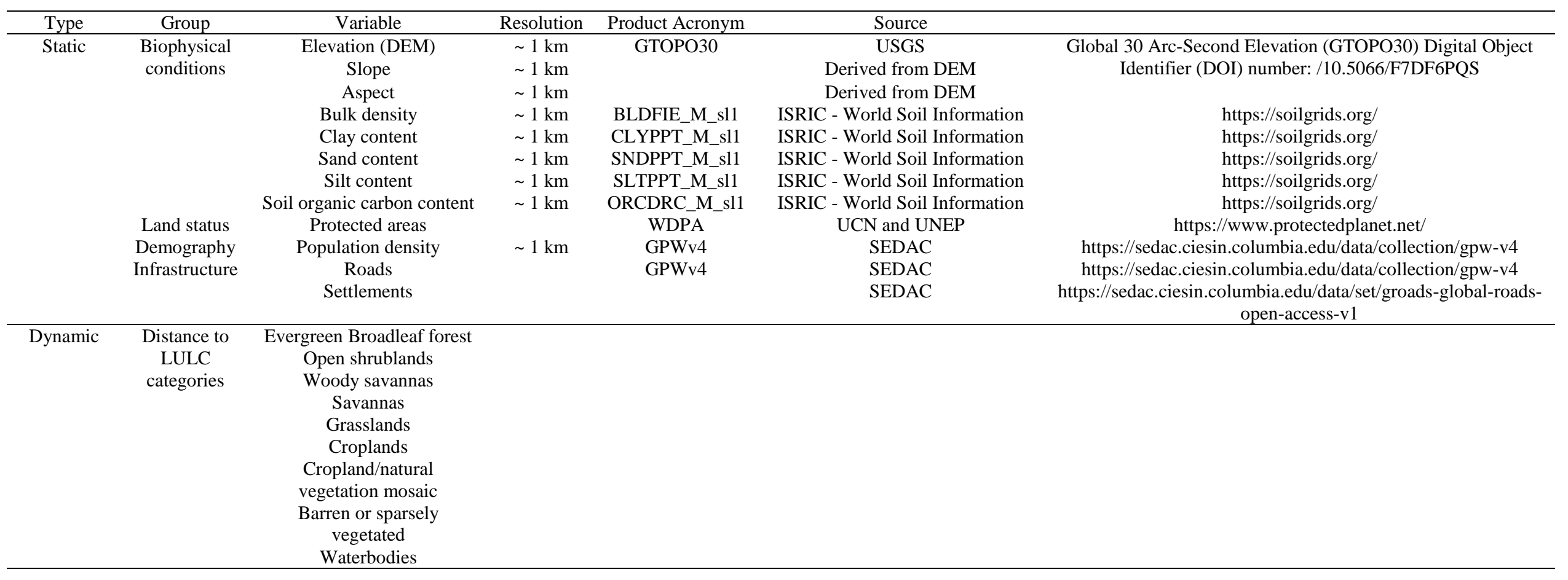


Table S12. Comparison of Noah and Satellite Derived Albedo values for MODIS-IGBP categories

\begin{tabular}{ccc}
\hline MODIS-IGBP Category & Noah & Houldcroft et al. (2009) \\
\hline 1 & 12 & 9.2 \\
2 & 12 & 13.9 \\
3 & $14-15$ & 10.3 \\
4 & $16-17$ & 13.3 \\
5 & $17-25$ & 11.2 \\
6 & $25-30$ & 13.4 \\
7 & $22-30$ & 16.1 \\
8 & $25-30$ & 13.1 \\
9 & 20 & 15.5 \\
10 & $19-23$ & 16.8 \\
11 & 14 & 10.2 \\
12 & $17-23$ & 16.5 \\
13 & 15 & 14.9 \\
14 & $18-23$ & 15.8 \\
\hline
\end{tabular}


Table S13. Noah-MP LAI Seasonal Profiles for each MODIS-IGBP Category

\begin{tabular}{ccccccccccccc}
\hline MODIS-IGBP Category & Jan. & Feb. & Mar. & Apr. & May & Jun. & Jul. & Aug. & Sep. & Oct. & Nov. & Dec. \\
\hline 1 & 4.0 & 4.0 & 4.0 & 4.0 & 4.0 & 4.0 & 4.0 & 4.0 & 4.0 & 4.0 & 4.0 & 4.0 \\
2 & 4.5 & 4.5 & 4.5 & 4.5 & 4.5 & 4.5 & 4.5 & 4.5 & 4.5 & 4.5 & 4.5 & 4.5 \\
3 & 0.0 & 0.0 & 0.0 & 0.6 & 1.2 & 2.0 & 2.6 & 1.7 & 1.0 & 0.5 & 0.2 & 0.0 \\
4 & 0.0 & 0.0 & 0.3 & 0.9 & 2.2 & 3.5 & 3.5 & 2.5 & 0.9 & 0.3 & 0.0 & 0.0 \\
5 & 2.0 & 2.0 & 2.2 & 2.6 & 3.5 & 4.3 & 4.3 & 3.7 & 2.6 & 2.2 & 2.0 & 2.0 \\
6 & 0.0 & 0.0 & 0.3 & 0.9 & 2.2 & 3.5 & 3.5 & 2.5 & 0.9 & 0.3 & 0.0 & 0.0 \\
7 & 0.0 & 0.0 & 0.2 & 0.6 & 1.5 & 2.3 & 2.3 & 1.7 & 0.6 & 0.2 & 0.0 & 0.0 \\
8 & 0.2 & 0.2 & 0.4 & 1.0 & 2.4 & 4.1 & 4.1 & 2.7 & 1.0 & 0.4 & 0.2 & 0.2 \\
9 & 0.3 & 0.3 & 0.5 & 0.8 & 1.8 & 3.6 & 3.8 & 2.1 & 0.9 & 0.5 & 0.3 & 0.3 \\
10 & 0.4 & 0.5 & 0.6 & 0.7 & 1.2 & 3.0 & 3.5 & 1.5 & 0.7 & 0.6 & 0.5 & 0.4 \\
11 & 0.2 & 0.3 & 0.3 & 0.5 & 1.5 & 2.9 & 3.5 & 2.7 & 1.2 & 0.3 & 0.3 & 0.2 \\
12 & 0.0 & 0.0 & 0.0 & 0.0 & 1.0 & 2.0 & 3.0 & 3.0 & 1.5 & 0.0 & 0.0 & 0.0 \\
13 & 0.0 & 0.0 & 0.0 & 0.0 & 0.0 & 0.0 & 0.0 & 0.0 & 0.0 & 0.0 & 0.0 & 0.0 \\
14 & 0.2 & 0.3 & 0.3 & 0.4 & 1.1 & 2.5 & 3.2 & 2.2 & 1.1 & 0.3 & 0.3 & 0.2 \\
15 & 0.0 & 0.0 & 0.0 & 0.0 & 0.0 & 0.0 & 0.0 & 0.0 & 0.0 & 0.0 & 0.0 & 0.0 \\
16 & 0.0 & 0.0 & 0.0 & 0.0 & 0.0 & 0.0 & 0.0 & 0.0 & 0.0 & 0.0 & 0.0 & 0.0 \\
17 & 0.0 & 0.0 & 0.0 & 0.0 & 0.0 & 0.0 & 0.0 & 0.0 & 0.0 & 0.0 & 0.0 & 0.0 \\
18 & 1.0 & 1.0 & 1.1 & 1.3 & 1.7 & 2.1 & 2.1 & 1.8 & 1.3 & 1.1 & 1.0 & 1.0 \\
19 & 0.6 & 0.6 & 0.7 & 0.8 & 1.2 & 1.8 & 1.8 & 1.3 & 0.8 & 0.7 & 0.6 & 0.6 \\
20 & 0.0 & 0.0 & 0.0 & 0.0 & 0.0 & 0.0 & 0.0 & 0.0 & 0.0 & 0.0 & 0.0 & 0.0 \\
\hline
\end{tabular}


Table S14. Noah LAI Maximum and Minimum Values for each MODIS-IGBP Category

\begin{tabular}{ccc}
\hline MODIS-IGBP Category & Min. & Max. \\
\hline Evergreen Needle Leaf Forest & 5.00 & 6.49 \\
Evergreen Broad Leaf Forest & 3.08 & 6.48 \\
Deciduous Needle Leaf Forest & 1.00 & 5.16 \\
Deciduous Broad Leaf Forest & 1.85 & 3.31 \\
Mixed Forest & 2.80 & 5.50 \\
Closed Shrubland & 0.50 & 3.66 \\
Open Shrubland & 0.60 & 2.60 \\
Woody Savanna & 0.50 & 3.66 \\
Savannas & 0.50 & 3.66 \\
Grasslands & 0.52 & 2.90 \\
Permanent Wetlands & 1.75 & 5.72 \\
Cropland & 1.56 & 5.68 \\
Urban and Built & 1.00 & 1.00 \\
Cropland/Natural Vegetation & 2.29 & 4.29 \\
Mosaic & & \\
Ice & 0.01 & 0.01 \\
Water & 0.10 & 0.75 \\
Barren/Sparsely Vegetated & 0.01 & 0.01 \\
Wooded Tundra & 0.41 & 3.35 \\
Mixed Tundra & 0.41 & 3.35 \\
Barren Tundra & 0.41 & 3.35 \\
\hline
\end{tabular}


Table S15: Annual Average Surface Sensible Heat Flux $\left(\mathrm{W} \mathrm{m}^{-2}\right)$ in WRF Grid Cells that experience LULCCs between 2001 and 2015

\begin{tabular}{ccccc}
\hline Transition & Noah & Noah-MP & CLM-D & CLM-AF \\
\hline Agricultural Expansion $^{*}$ & 2.35 & -9.14 & -3.37 & 0.94 \\
10 to 12 & -0.22 & -1.53 & 0.22 & 0.67 \\
2 to 14 & 0.51 & 1.29 & 4.67 & 5.18 \\
8 to 14 & 5.03 & -14.38 & -5.85 & 0.12 \\
10 to 14 & -0.91 & -0.90 & 0.42 & 4.42 \\
\hline Deforestation/Degradation* $^{*}$ & 4.08 & -3.38 & -2.03 & -2.12 \\
8 to 9 & 10.45 & -3.66 & -1.94 & -1.45 \\
9 to 7 & -10.14 & -0.19 & -1.96 & -5.81 \\
9 to 10 & -1.95 & 0.12 & -5.23 & -3.34 \\
\hline Greening* & 5.65 & 0.12 & 2.16 & 2.13 \\
9 to 8 & -6.83 & 3.07 & 0.46 & 0.54 \\
10 to 9 & 3.08 & 11.27 & 5.45 & 4.39 \\
16 to 7 & 19.58 & 0.77 & 9.60 & 3.44 \\
16 to 10 & 24.75 & -2.13 & 4.45 & 11.34 \\
\hline
\end{tabular}

*: Shows average difference for a broad class of LULCC followed by the average difference in the major MODIS LULC transitions that comprise that class. MODIS Land Use Categories: 2 Evergreen Broad Leaf Forest; 7 - Open Shrublands; 8 - Woody Savanna; 9 - Savannas; 10 Grasslands; 12 - Croplands; 14 -Cropland/Natural Mosaic; 16 - Barren/ Sparsely Vegetated 
Table S16: Annual Average Surface Latent Heat Flux $\left(\mathrm{W} \mathrm{m}^{-2}\right)$ in WRF Grid Cells that experience LULCCs between 2001 and 2015

\begin{tabular}{ccccc}
\hline Transition & Noah & Noah-MP & CLM-D & CLM-AF \\
\hline Agricultural Expansion $^{*}$ & 2.26 & -1.67 & -3.87 & -3.82 \\
10 to 12 & 1.01 & 0.30 & -2.11 & -2.75 \\
2 to 14 & -11.72 & -18.41 & -27.82 & -25.57 \\
8 to 14 & 9.33 & 0.28 & -1.68 & -3.80 \\
10 to 14 & 1.39 & 0.81 & -0.65 & -2.28 \\
\hline Deforestation/Degradation* $^{*}$ & 1.61 & -1.22 & -1.46 & -3.70 \\
8 to 9 & 5.37 & -0.13 & -0.38 & -2.93 \\
9 to 7 & -3.83 & -1.85 & 0.37 & -4.57 \\
9 to 10 & -0.29 & -0.60 & -1.04 & -1.86 \\
\hline Greening* & 0.14 & 4.76 & 7.81 & 5.83 \\
9 to 8 & -9.46 & 0.41 & 2.94 & 3.33 \\
10 to 9 & -0.59 & -0.34 & -1.05 & -0.76 \\
16 to 7 & 2.66 & 3.94 & 6.68 & 2.18 \\
16 to 10 & 8.04 & 5.18 & 8.13 & 4.79 \\
\hline
\end{tabular}

*: Shows average difference for a broad class of LULCC followed by the average difference in the major MODIS LULC transitions that comprise that class. MODIS Land Use Categories: 2 Evergreen Broad Leaf Forest; 7 - Open Shrublands; 8 - Woody Savanna; 9 - Savannas; 10 Grasslands; 12 - Croplands; 14 -Cropland/Natural Mosaic; 16 - Barren/ Sparsely Vegetated 


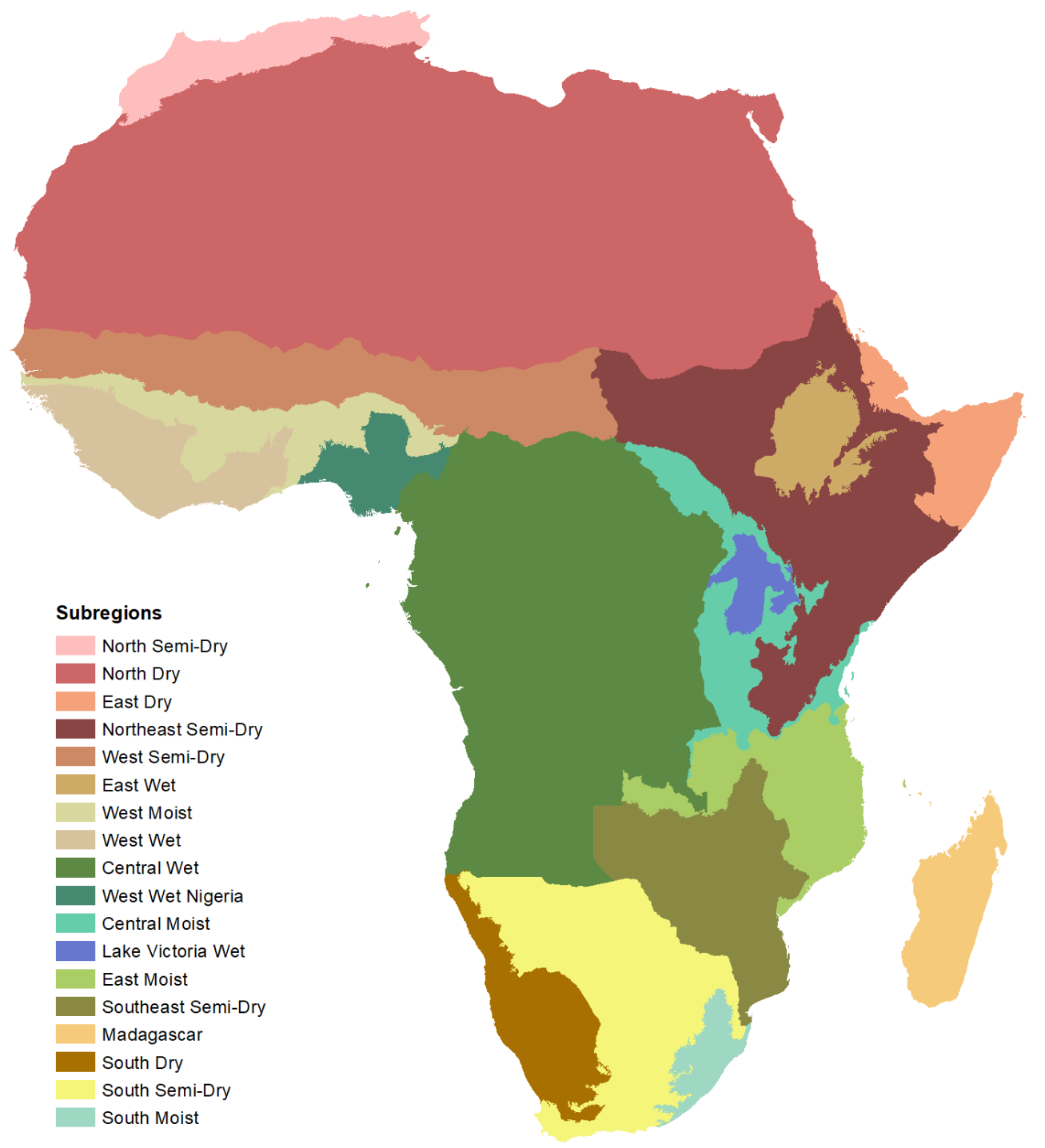

Fig S1. Regions used to model LULCC in Africa 

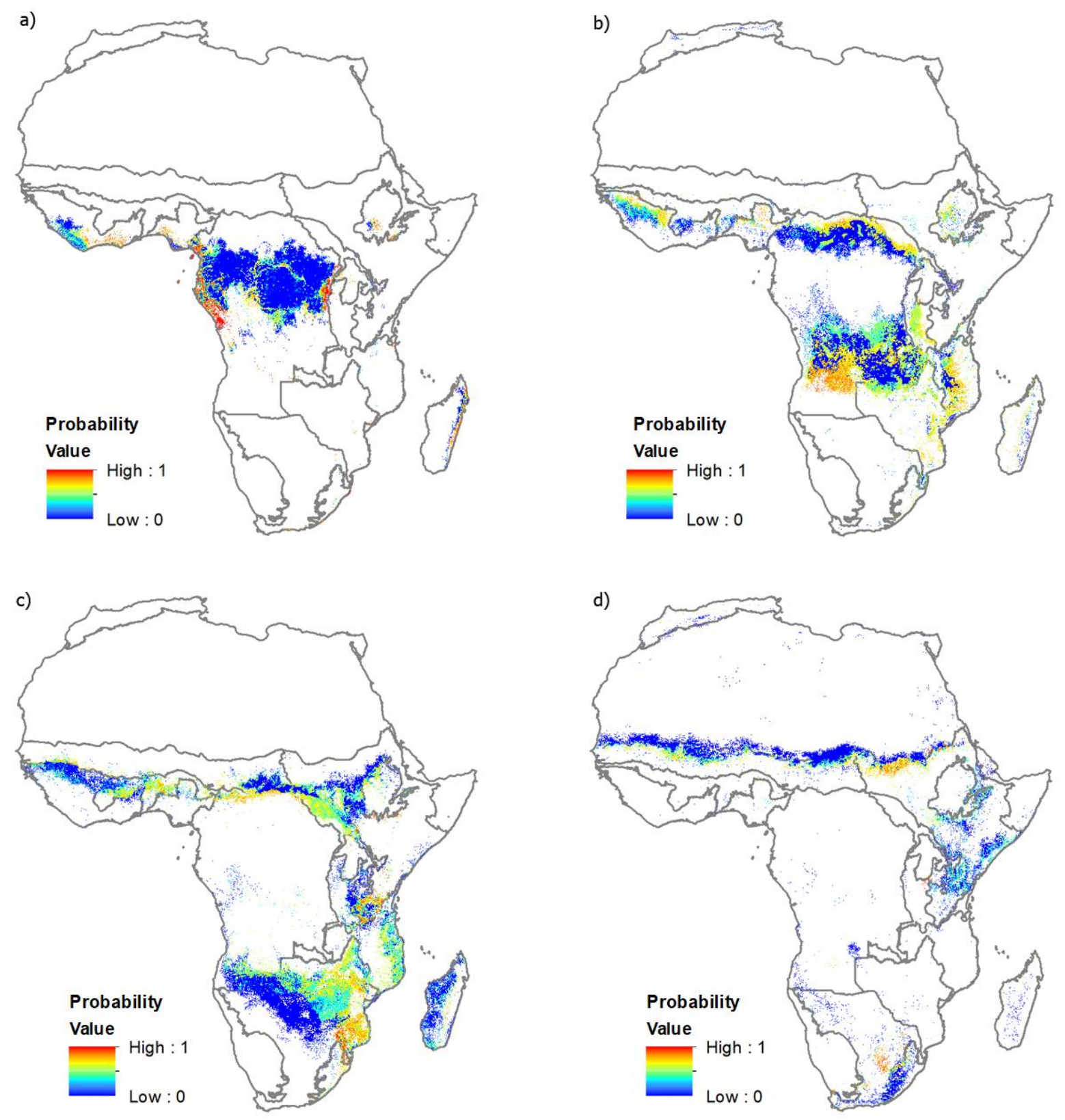

Fig S2. Probability maps for transitions a) Evergreen broadleaf forest to Cropland/Natural vegetation mosaic, b) Woody savannas to savannas, c) Savannas to woody savannas, and d) Grasslands to croplands. 


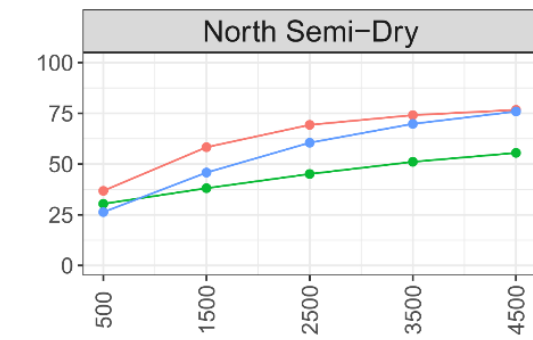

$\rightarrow$ BSV to OShr - Gr to OShr $\rightarrow$ OShr to $\mathrm{Cr}$

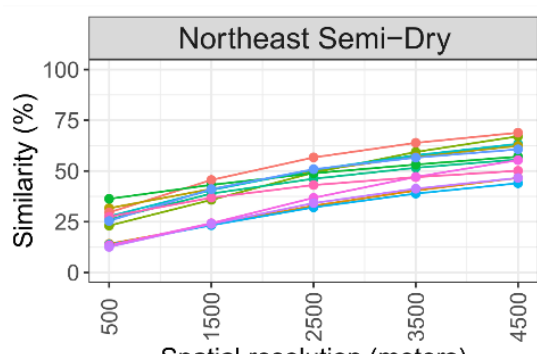

-BSV to OSh - Cr to Gr $\rightarrow \mathrm{Cr} / \mathrm{NM}$ to $\mathrm{Cr}$ - $\mathrm{Cr} / \mathrm{VM}$ to $\mathrm{Gr}$ - Cr/vM to Sav - $\mathrm{Gr}$ to $\mathrm{Cr}$ $\rightarrow-G r$ to OSh - OShr to $\mathrm{Gr}$ $\rightarrow$ Sav to $\mathrm{Cr}$ - Sav to $\mathrm{Cr} / \mathrm{NM}$ - Sav to Gr -WSav to Sav

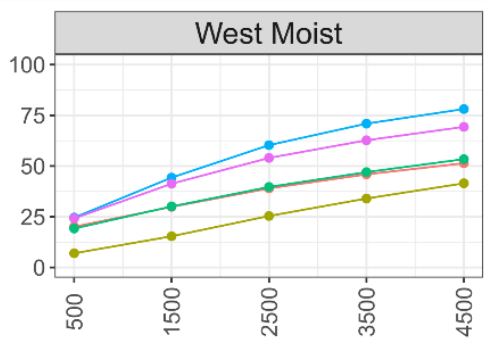

\section{- Gr to $\mathrm{Cr} / \mathrm{NM}$} $\rightarrow$ Sav to Cr/VM $\rightarrow$ WSav to $\mathrm{Cr}$ -WSav to CrNM -WSar to Sav

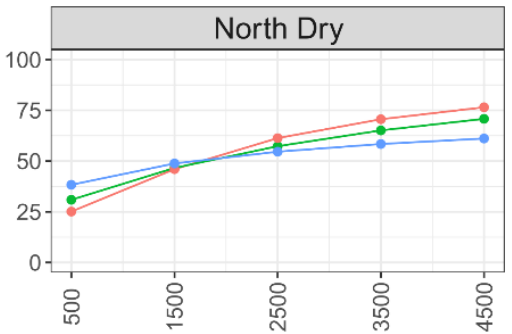

$-\mathrm{BSV}$ to Gr - CrNM to Gr - OShr to BSV

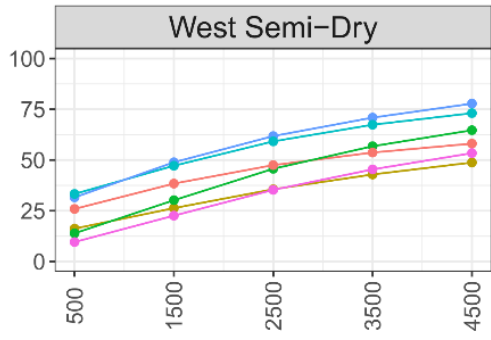

$\rightarrow \mathrm{Cr} / \mathrm{NM}$ to $\mathrm{Gr}$ $-\mathrm{Gr}$ to $\mathrm{Cr}$ - $\mathrm{Gr}$ to $\mathrm{Cr} / \mathrm{MM}$ - OShr to CrNM - OShr to Gr $\rightarrow$ Sav to $\mathrm{Cr} / \mathrm{VM}$

- Cr/VM to WSav $\rightarrow$ EBF to $\mathrm{Cr} / \mathrm{VM}$

- Sav to WSav -WSav to Sav

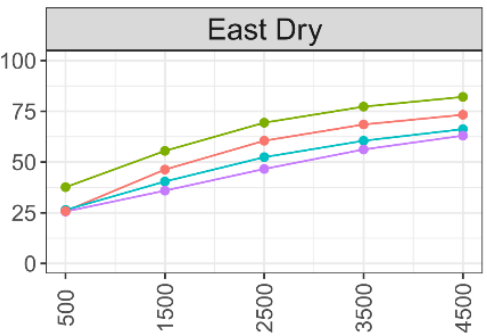

- BSV to OShr - Cr/VM to OShr $\rightarrow$ Gr to BSV $\rightarrow$ Gr to OShr

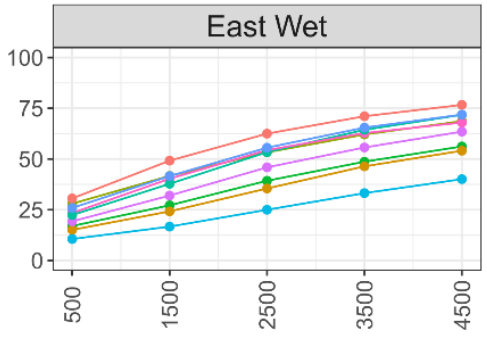

- CrNM to C - CrNMM to WSav - $\mathrm{Gr}$ to $\mathrm{Cr}$ - Gr to $\mathrm{Cr} / \mathrm{NM}$ - Sav to $\mathrm{Cr}$ - Sav to Cr/NM - Sav to WSav -WSav to $\mathrm{Cr} / \mathrm{NM}$ -WSav to Sav
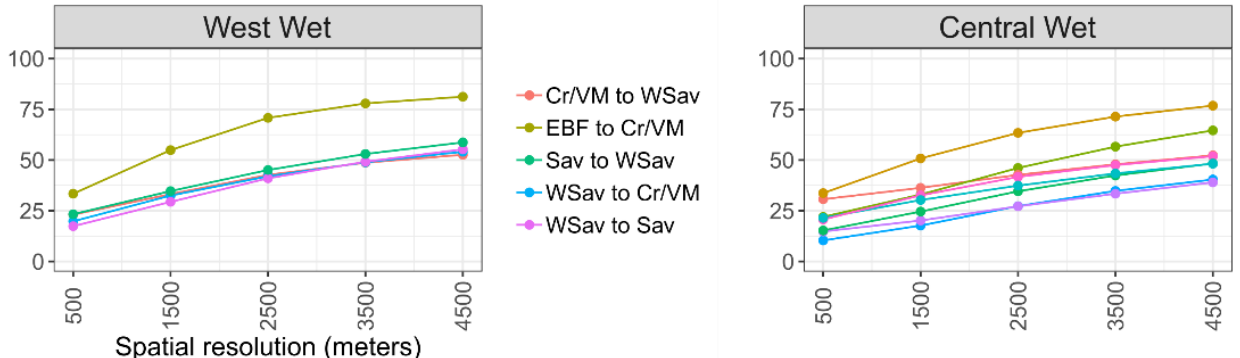

- Cr to WSar - Cr/NM to WSav -PWet to EBF - Sav to CrNM - Sar to WSar $\rightarrow$ WSav to Cr/NM -WSav to EBF

-WSav to Sav

Fig S3 Part 1. Fuzzy similarity index between real and simulated changes for each transition by region.

Acronyms: $\mathrm{EBF}=$ Evergreen Broadleaf forest, $\mathrm{MF}=$ Mixed forest, $\mathrm{CShr}=$ Closed shrublands, OShr $=$ Open shrublands, $\mathrm{WSav}=$ Woody savannas, $\mathrm{Sav}=$ Savannas, $\mathrm{Gr}=$ Grasslands, $\mathrm{PWet}=$ Permanent wetlands, $\mathrm{Cr}=$ Croplands, $\mathrm{Cr} / \mathrm{VM}=\mathrm{Cropland} / \mathrm{Natural}$ vegetation mosaic, $\mathrm{BSV}=$ Barren or sparsely vegetated. 

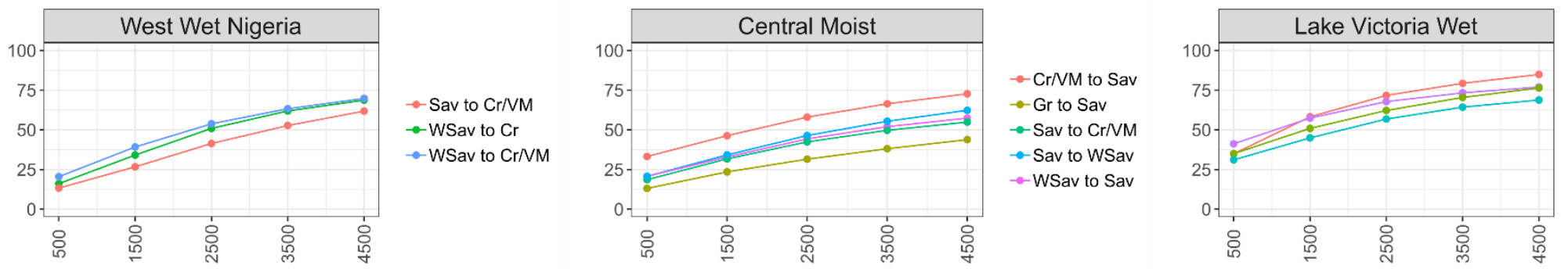

- EBF to $\mathrm{Cr} / \mathrm{NM}$ $\rightarrow$ Sav to CrNM - Sav to WSav

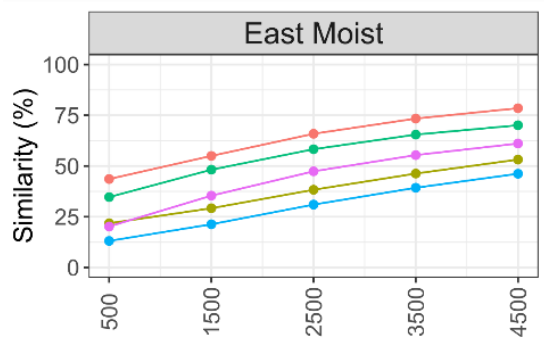

$-\mathrm{Cr} / \mathrm{VM}$ to Sav -MF to Sav - MF to WSar - Sav to WSav

-WSav to Sav
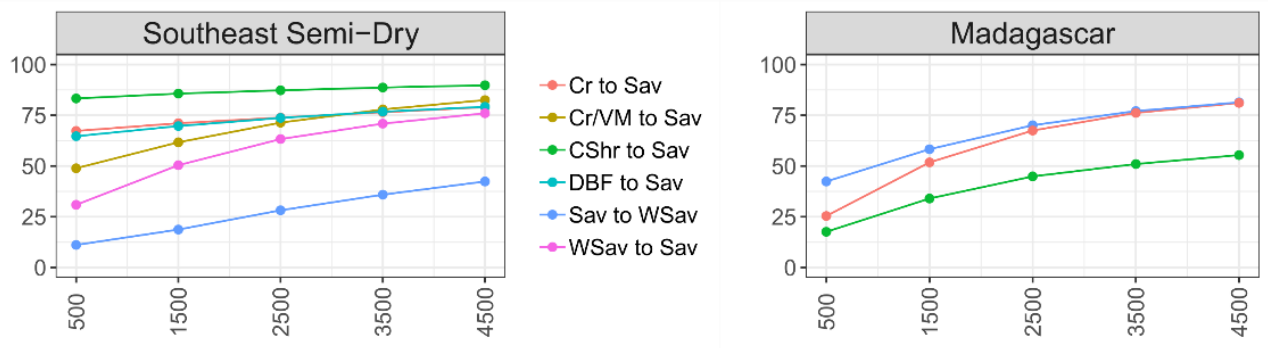

- Sav to OShr - Sav to WSav -WSav to Sav
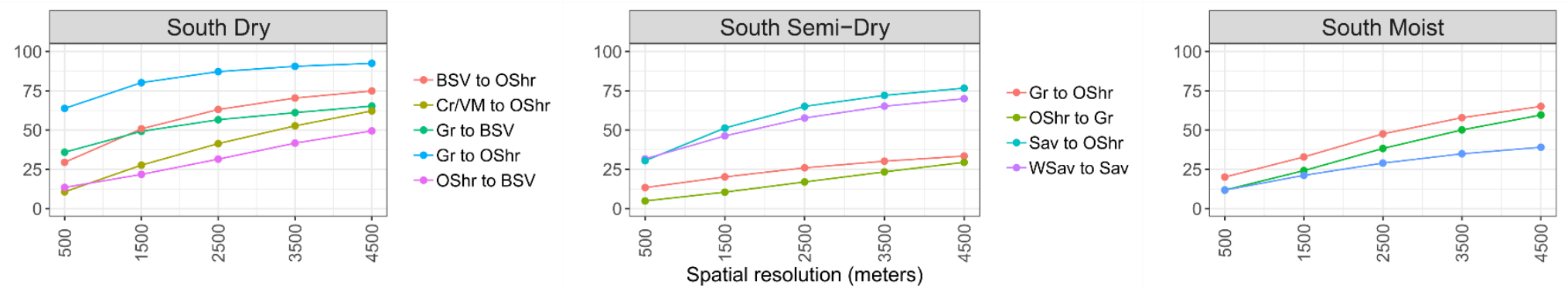

- $\mathrm{Cr}$ to $\mathrm{Gr}$ - CrNM to Sav $\rightarrow$ OShr to Gr

Fig S3 Part 2. Fuzzy similarity index between real and simulated changes for each transition by region.

Acronyms: $\mathrm{EBF}=$ Evergreen Broadleaf forest, $\mathrm{MF}=$ Mixed forest, $\mathrm{CShr}=$ Closed shrublands, OShr $=$ Open shrublands, WSav = Woody savannas, Sav = Savannas, $\mathrm{Gr}=$ Grasslands, $\mathrm{PWet}=$ Permanent wetlands, $\mathrm{Cr}=$ Croplands, $\mathrm{Cr} / \mathrm{VM}=\mathrm{Cropland} / \mathrm{Natural}$ vegetation mosaic, $\mathrm{BSV}=$ Barren or sparsely vegetated. 


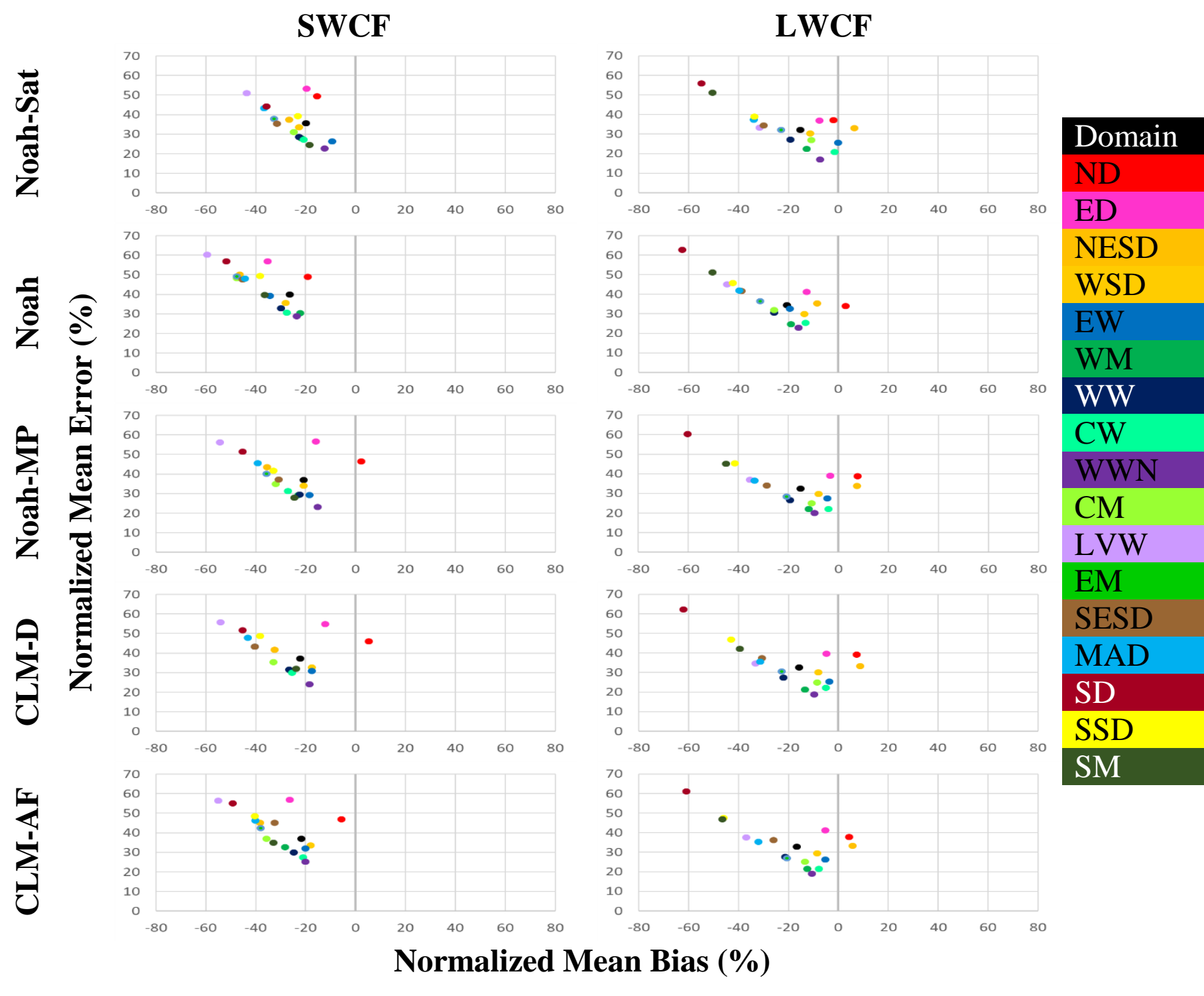

Fig S4

plot of domain and regional WRF SWCF and LWCF model performance statistics verses CERES-EBAF estimates 


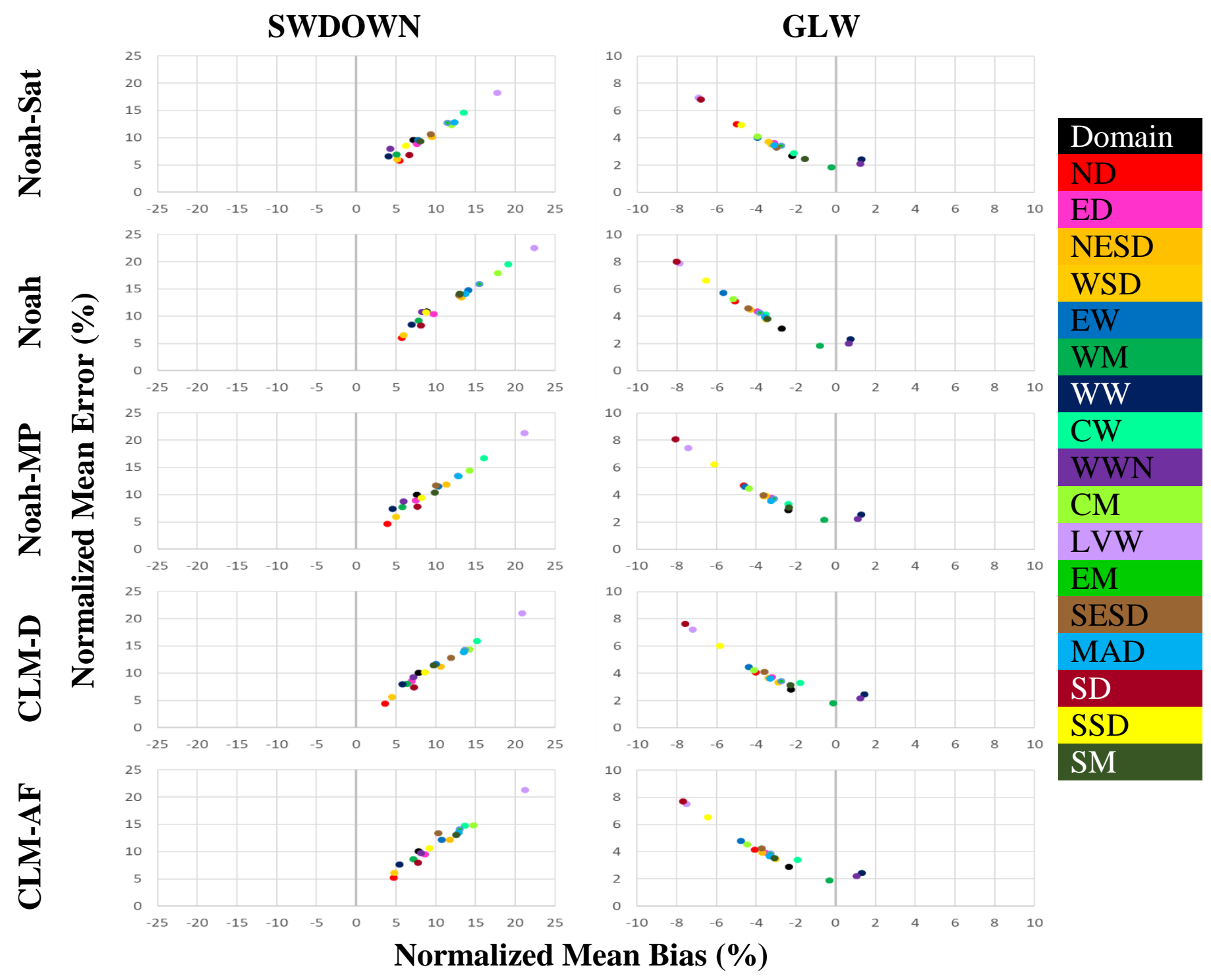

Fig S5: Soccer plot of domain and regional WRF SWDOWN and GLW model performance statistics verses CERES-EBAF estimates 


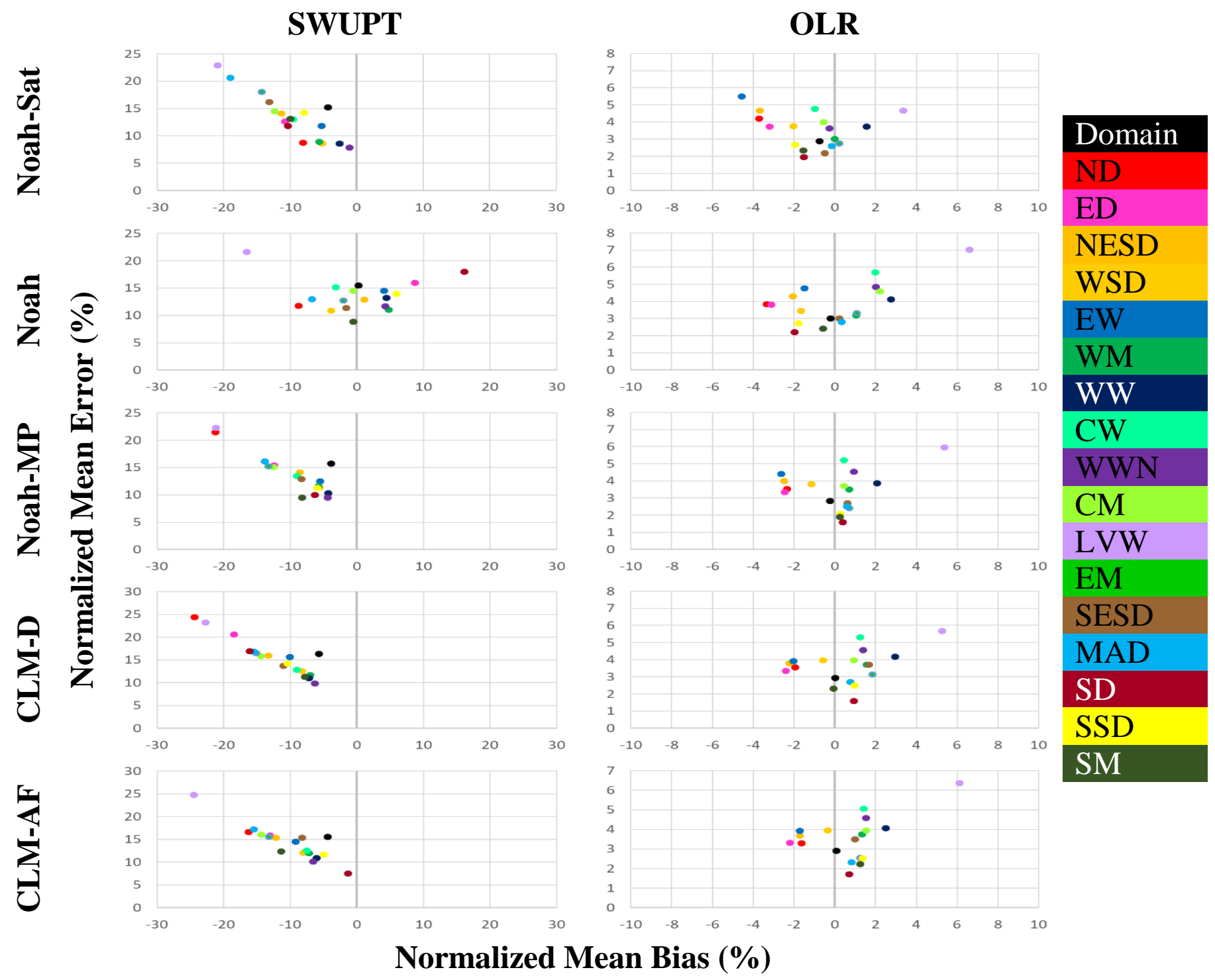

Fig S6: Soccer plot of domain and regional WRF SWUPT and OLR model performance statistics verses CERES-EBAF estimates 
T2MAX

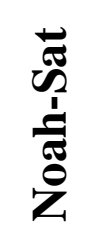

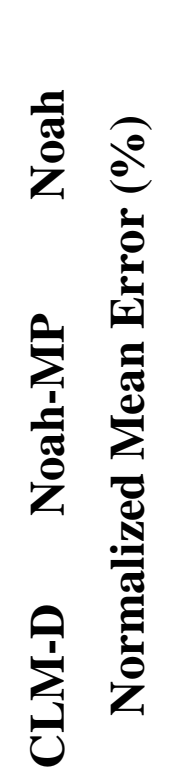

$\sum_{0}^{4}$
T2MIN
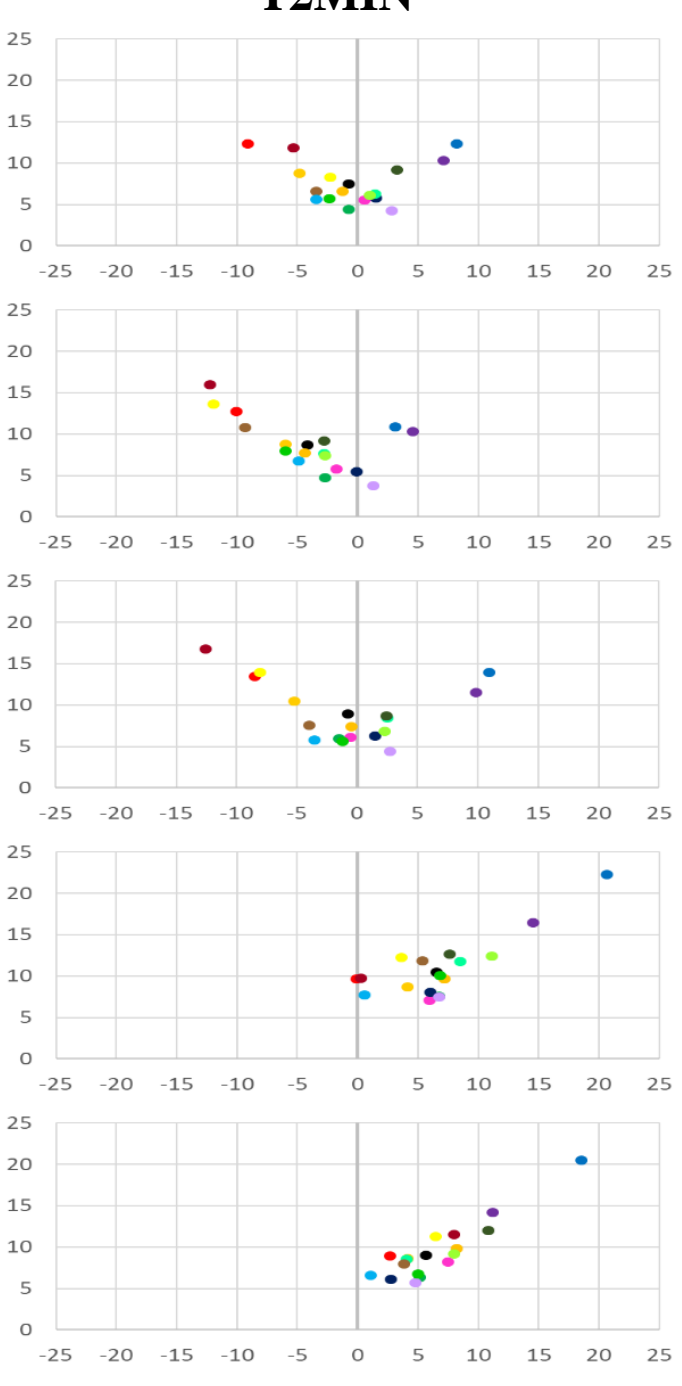

Normalized Mean Bias $(\%)$
DTR
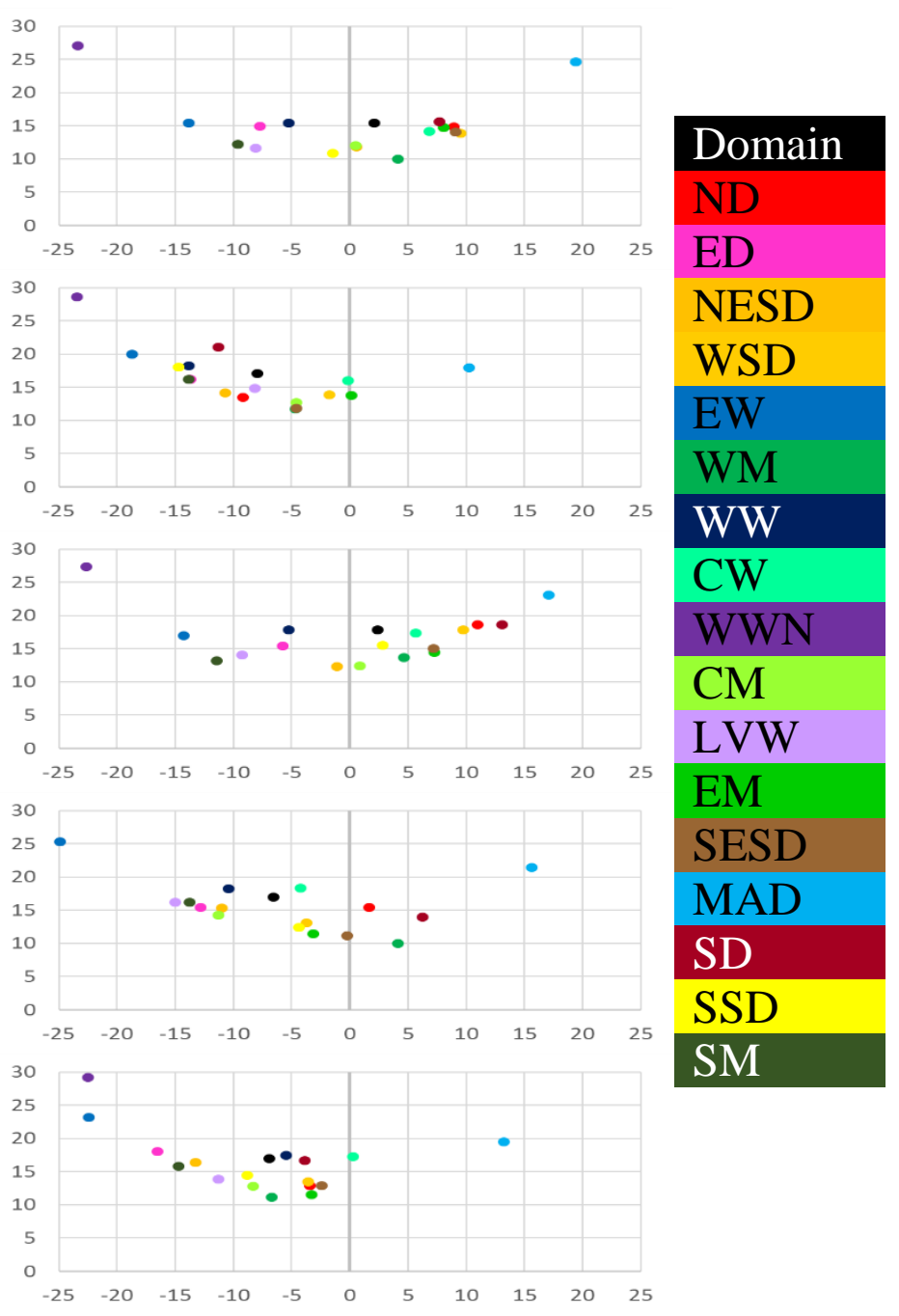

Fig S7. Soccer plots of domain and regional WRF annual average daily maximum 2-m temperature, daily minimum 2-m temperature, and diurnal temperature range compared to CRU estimates from the five WRF LSM configuration 

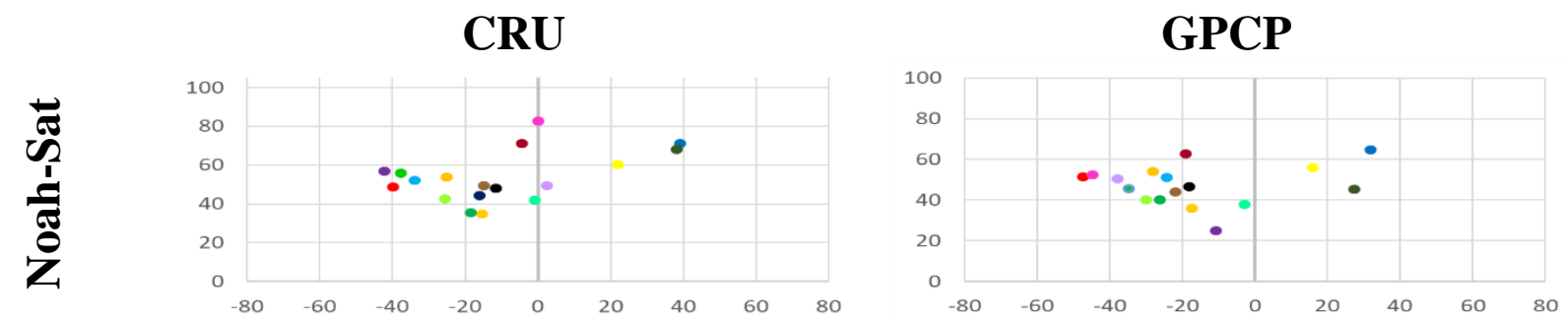

Domain
ND
ED
NESD
WSD
EW
WM
WW
CW
WWN
CM
LVW
EM
SESD
MAD
SD
SSD
SM
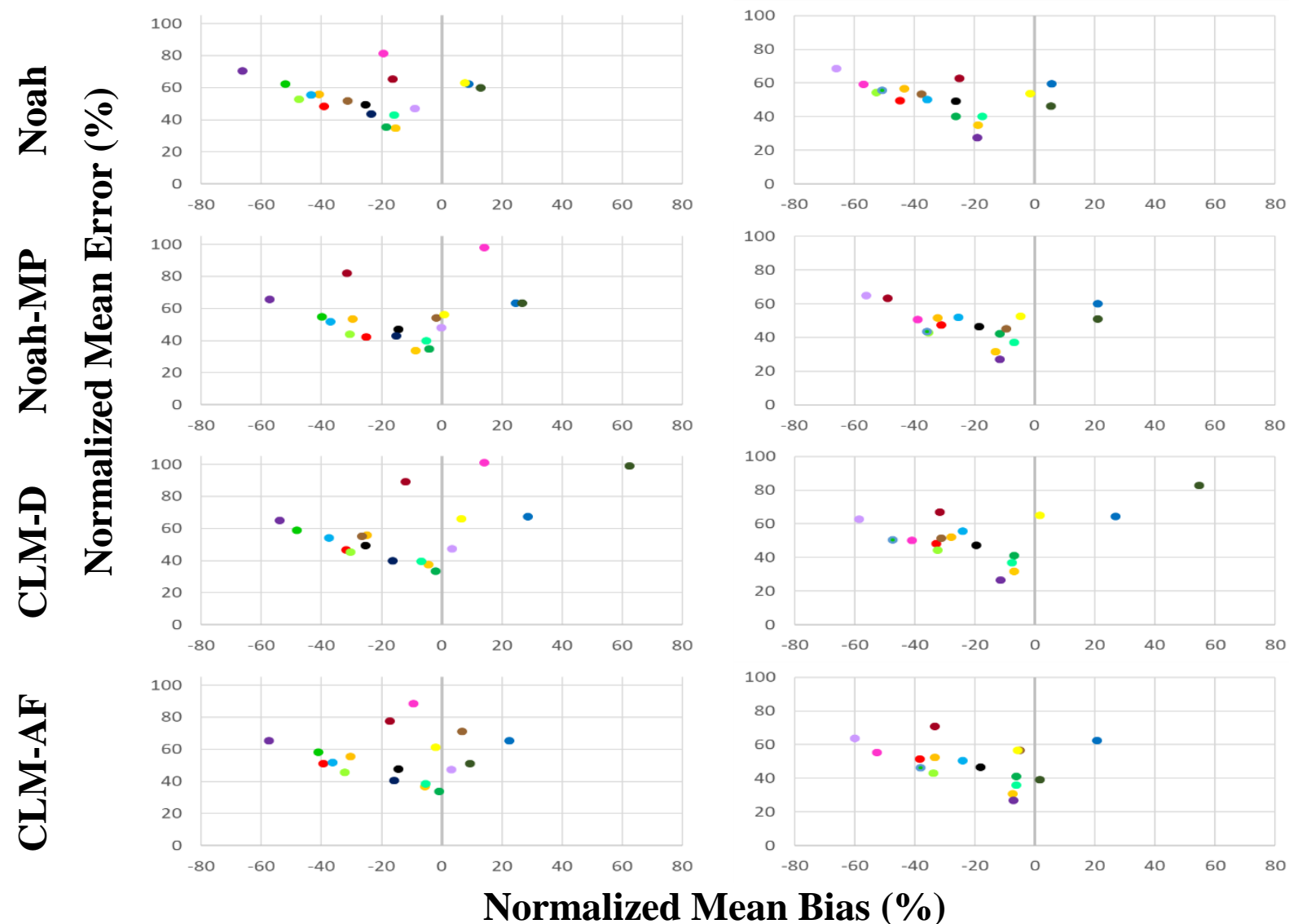

Normalized Mean Bias (\%)

Fig S8: Soccer plot of domain and regional WRF precipitation model performance statistics verses CRU and GPCP estimates 


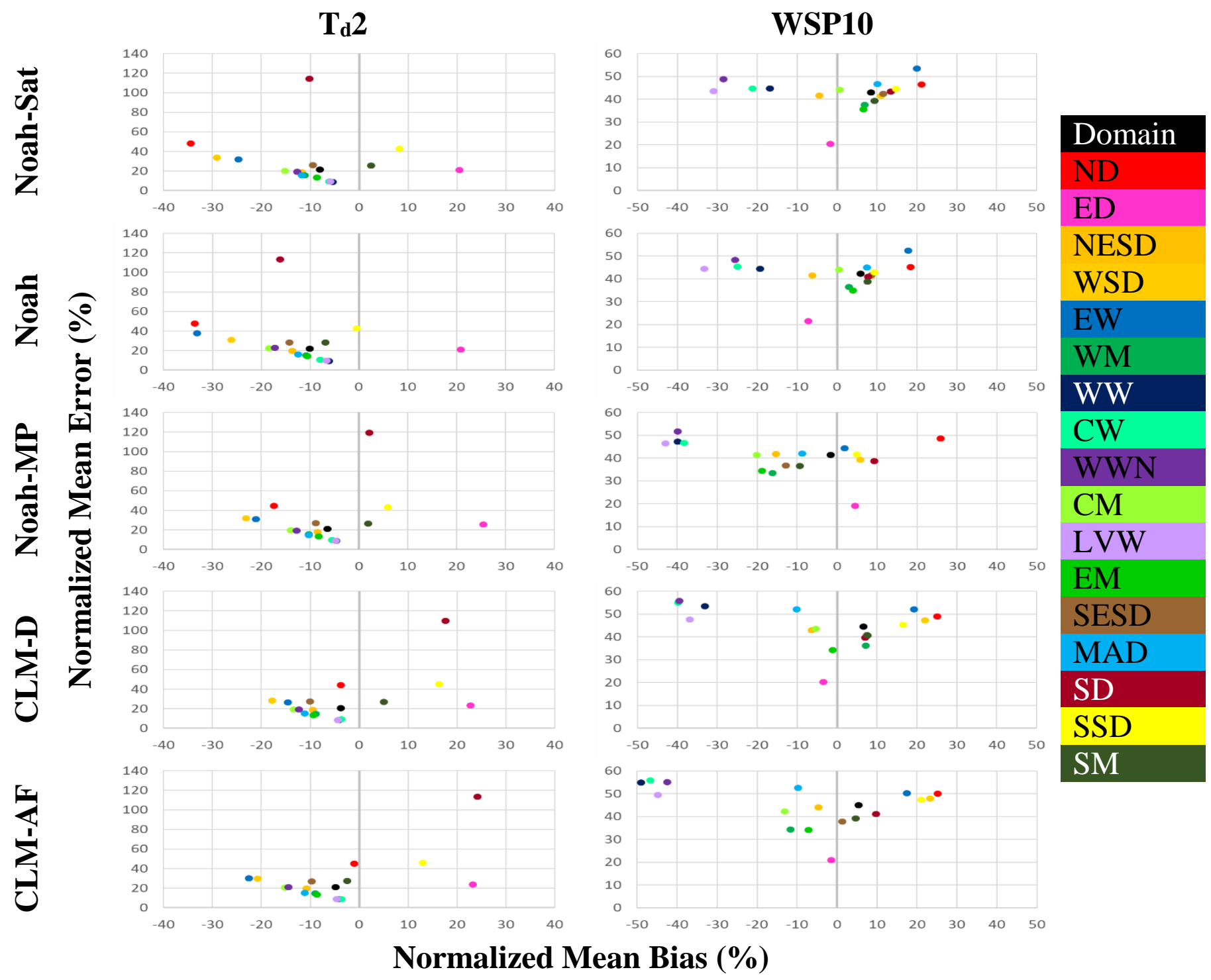

Fig S9: Soccer plot of domain and regional precipitation model performance statistics of WRF 2-m dew point temperature and 10-m wind speed verses NCDC-ISD observations 


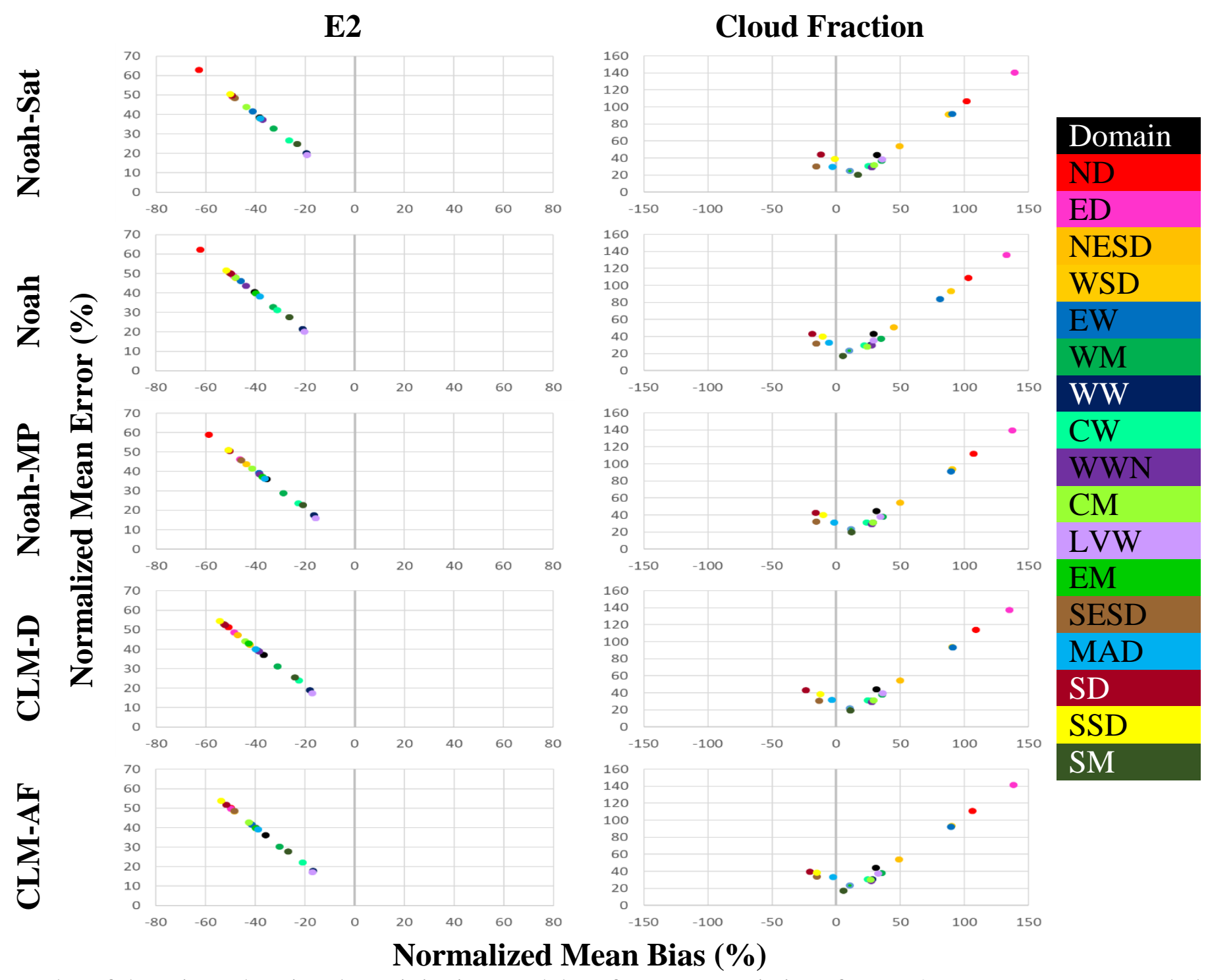

Fig S10: Soccer plot of domain and regional precipitation model performance statistics of WRF 2-m vapor pressure and cloud fraction speed verses CRU estimates 


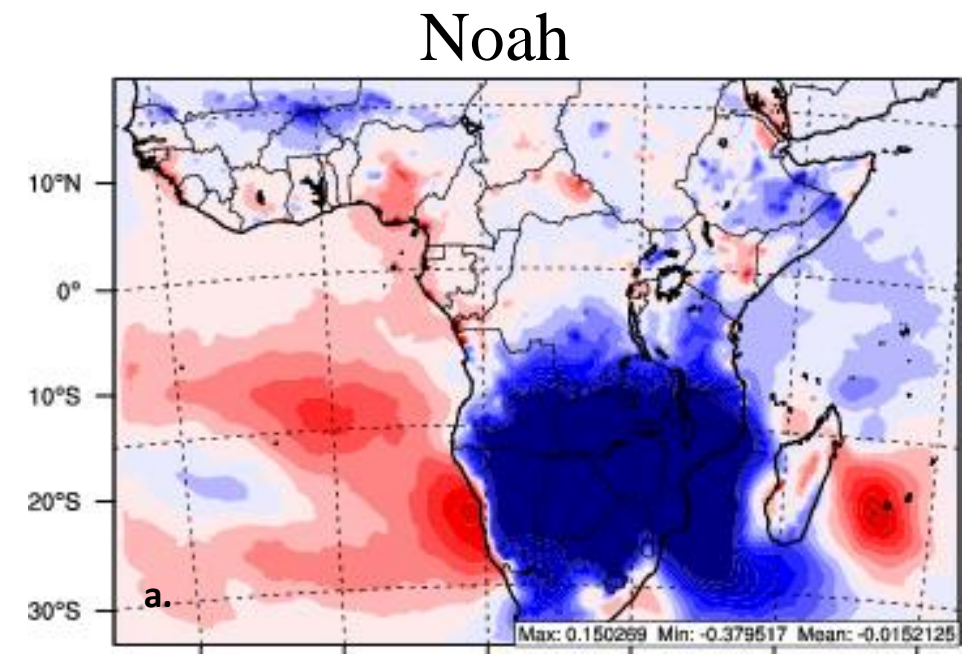

CLM-D
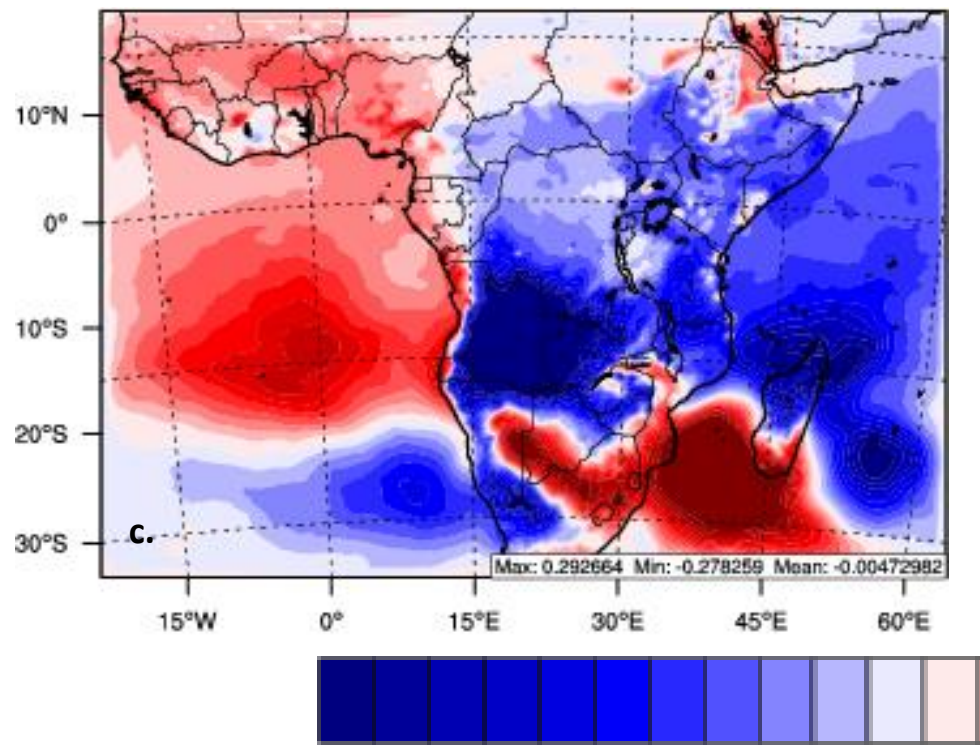

$\begin{array}{lllll}-0,1 & -0,08 & -0,06 & -0,04 & -0,02\end{array}$

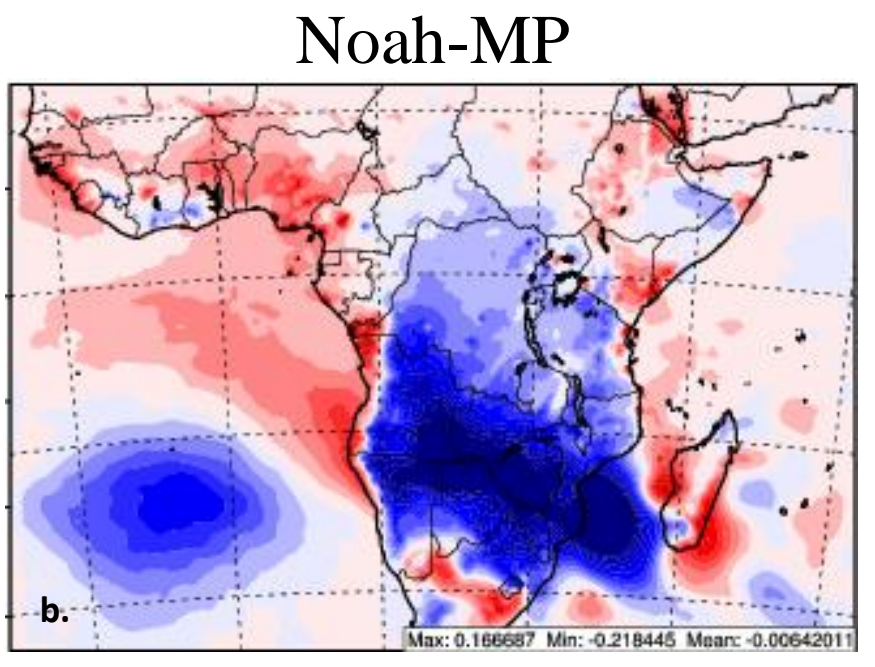

CLM-AF

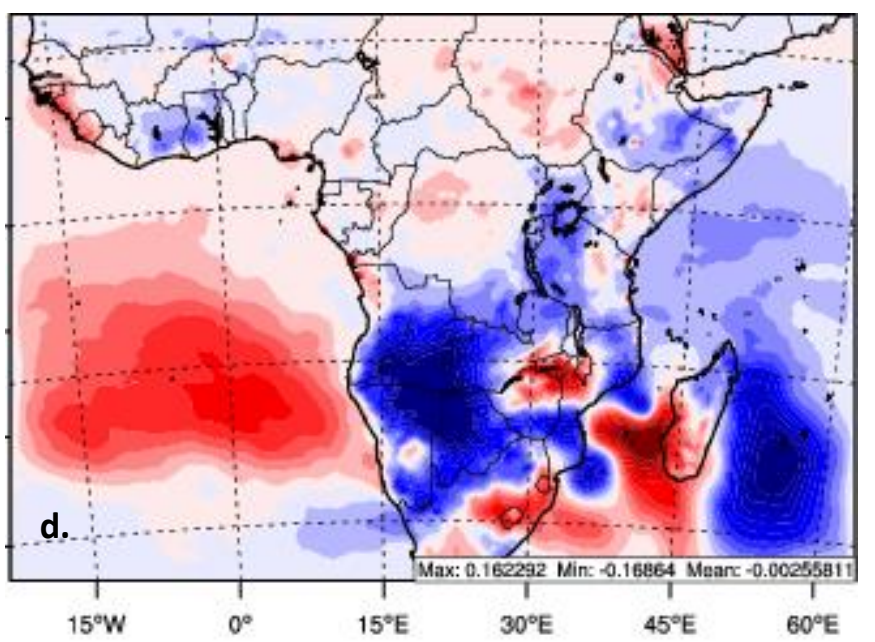

Fig S11. Differences in average JFD sea level pressure (hPa) between the dynamic and 2001 LULC simulations using (a) Noah, (b) Noah-MP, (c) CLM-D, and (d) CLM-AF 


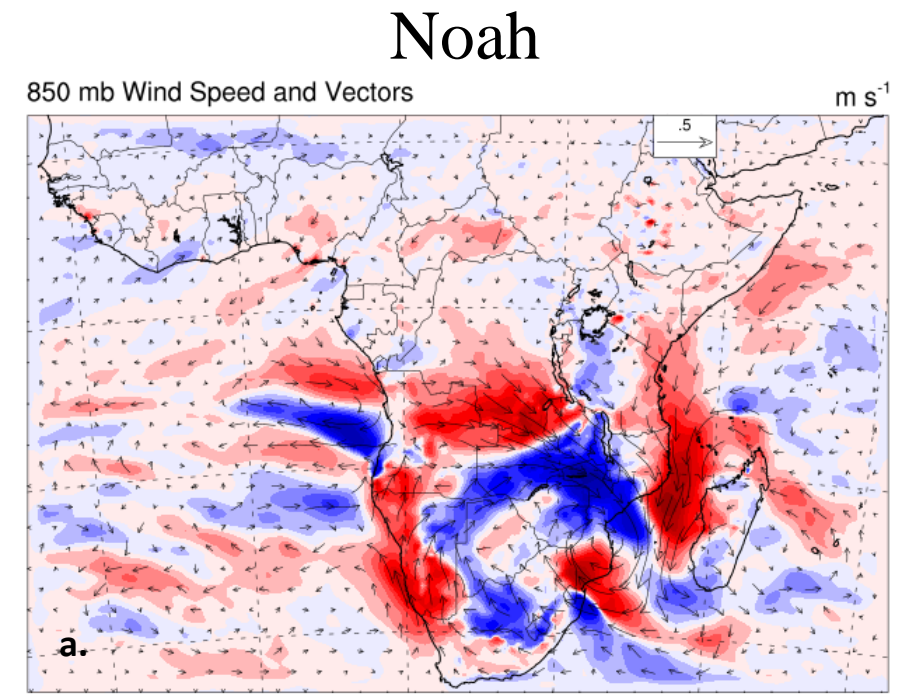

CLM-D

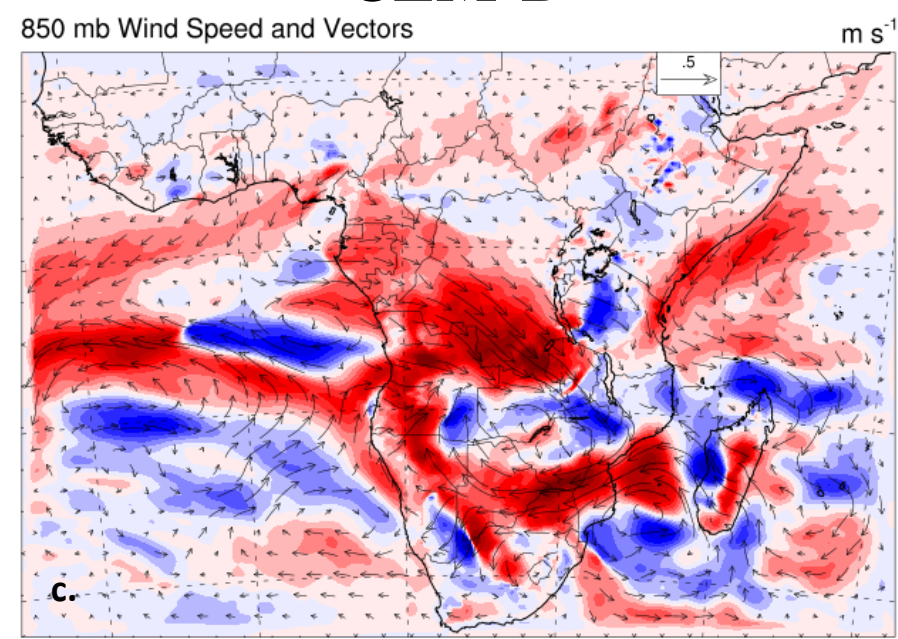

Noah-MP

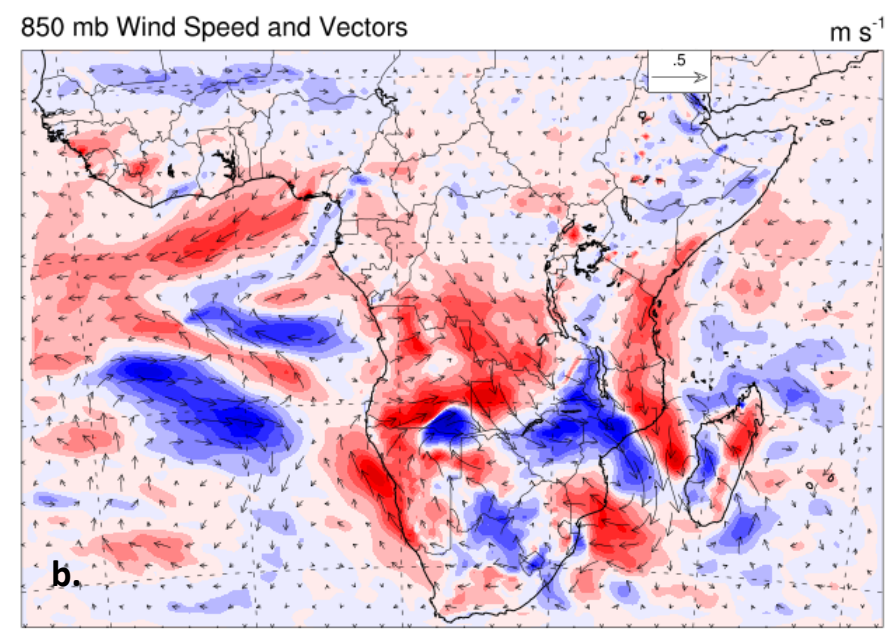

CLM-AF

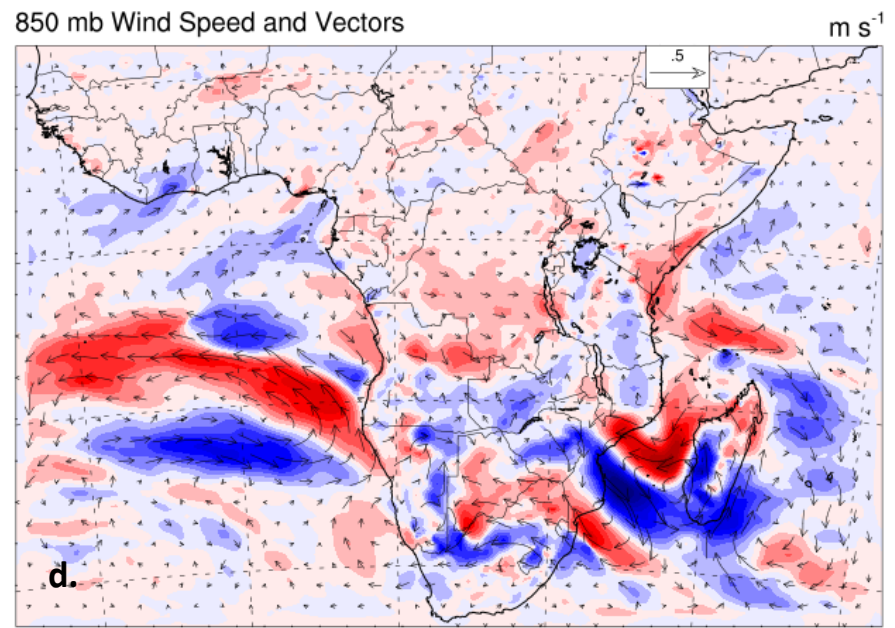

0.5

0.45

0.4

0.35

0.3

0.25

0.2

0.15

0.1

0.05

0

$-0.05$

$-0.1$

$-0.15$

$-0.2$

$-0.25$

$-0.3$

$-0.35$

$-0.4$

$-0.45$

$-0.5$

Fig S12. Differences in average JFD $850 \mathrm{mb}$ wind vectors and wind speed $\left(\mathrm{m} \mathrm{s}^{-1}\right)$ between the dynamic and 2001 LULC simulations using (a) Noah, (b) Noah-MP, (c) CLM-D, and (d) CLM-AF 